\title{
Multi-omics Reveal the Enabling Factors of Aplastic Anaemia
}

\author{
Xiaoyu Zhao \\ Tianjin Medical University \\ Chaomeng Wang \\ Tianjin Medical University \\ Chenchen Liu \\ Tianjin Medical University \\ Nan Jiang \\ Tianjin Medical University \\ Shaoxue Ding \\ Tianjin Medical University General Hospital \\ Yingying Chen \\ Tianjin Medical University \\ Liping Yang \\ Tianjin Medical University \\ Chunyan Liu \\ Tianjin Medical University General Hospital \\ Rong Fu ( $\square$ furong8369@tmu.edu.cn ) \\ Tianjin Medical University General Hospital
}

\section{Research Article}

Keywords: macrogene CG-MS, lipid metabolism, aplastic anaemia, Citrobacter, stearic acid, long-chain polyunsaturated fatty acids

Posted Date: February 4th, 2022

DOI: https://doi.org/10.21203/rs.3.rs-1313352/v1

License: @ (i) This work is licensed under a Creative Commons Attribution 4.0 International License. Read Full License 


\section{Abstract}

Background: Aplastic anemia is a kind of anemia caused by bone marrow failure due to autoimmune abnormalities. Numerous studies have shown that autoimmunity is regulated by intestinal microflora, and that most intestinal microflora regulates immunity through short chain fatty acids. At the same time, almost all patients with aplastic anemia will have bone marrow adipose tissue, and the process of bone marrow adipose tissue is regulated by medium and long chain fatty acids. Our previous studies have found that the intestinal flora of aplastic anemia patients is different from that of normal people. Therefore, this study aims to conduct a comprehensive detection of the intestinal flora and fatty acid metabolism in patients with aplastic anemia and to study their correlation.

Methods: Fatty acid compositions in the peripheral plasma and bone marrow supernatants of 12 newly diagnosed aplastic anaemia patients and 10 normal controls were comprehensively analysed by CG-MS. Gene sequencing of faecal samples from both groups was also performed with macrogene sequencing technology.

Results: Based on the metagenomic sequencing technology, we analyzed the difference of intestinal flora between group AA and group NC. We found that the main difference between the two groups existed in the bacterial community, and the abundance of most microbial species showed a downward trend. Based on the CG-MS lipid detection technique, we found that there were differences in lipid metabolism between the AA group and the NC group, whether short chain fatty acids or medium and long chain fatty acids. 8. The most attractive findings are interrelationships between the Citrobacter spp $\square$ stearic acid (c18:0), isobutyric acid, and lysine degradation and betaine biosynthesis pathway and the interrelationship between catenibacterium_mitsuokai species at Catenibacterium, cis-7,10,13,16-docosatetraenoic acid (c22:4), ubiquinone and other terpenoid quinones pathway.

We also found the intercorrelation between the abundance of Enhydrobacter genus and Enhydrobacter_aerosaccus species, cis-13,16-docosadienoic acid in peripheral plasma, cis-7,10,13,16-docosatetraenoic acid in bone marrow supernatant, tyrosine and tryptophan biosynthesis pathways.

Conclusions: Our results show that Citrobacter infection may be a driving factor for aplastic anaemia, identifying a potential role for stearic acid in the immunopathogenesis of aplastic anaemia. Our study also demonstrates the potential role of 22-carbon long-chain polyunsaturated fatty acids, which may not only be involved in the metabolism of fibroblasts in bone marrow but also influence the formation of the bone marrow microenvironment, in aplastic anaemia.

\section{Background}

Aplastic anaemia is a severe form of anaemia caused by bone marrow failure and is often associated with lipomatosis of the bone marrow. Almost all cases of acquired regenerative anaemia appear to be mediated by immunity ${ }^{1}$. The immunopathogenesis of aplastic anaemia has long been a hot topic in the field of the disease, but metabolic studies in patients with aplastic anaemia are rare. Previous studies, however, have shown that myelolipemia, a common condition in patients with aplastic anaemia, involves a variety of metabolites, especially fatty acids. Myelolipemia has been identified as a competitive process between bone marrow adipocytes and osteoblasts derived from a common mesenchymal stem cell; this process involves the Wnt/ $\beta$-catenin signalling pathway ${ }^{2}$, Wnt signalling pathway inhibitor DKK $1^{3}$, antagonist-associated secretory protein SFRP1 (Bodine et al., 2004 ), and paracrine and hormonal signalling pathways. Omega-3 unsaturated fatty acids and palmitic acid inhibit the differentiation of fibroblasts into osteoblasts by regulating these signalling pathways ${ }^{4}$. Saturated fatty acids produced by bone marrow adipocytes have a positive feedback effect on bone marrow adipogenesis by inhibiting osteoblast function and promoting adipocyte synthesis ${ }^{5}$. Palmitic acid can reduce the expression of RANKL, DKK1 and sclerosing protein in osteocytes and induce the apoptosis of osteoblasts, thus reducing the survival rate of bone cells ${ }^{6}$. Thus, we hypothesize that fatty acid metabolism and the process of bone marrow steatosis derived from AA are inextricably linked. What is not clear, however, is the change in fatty acid metabolism in aplastic anaemia patients. The correlation between intestinal microflora and lipid metabolism has been increasingly recognized. First, gut microbes are involved in short-chain fatty acid production and metabolism ${ }^{7}$. In the intestine, Akkermansia muciniphila metabolizes propionic acid ${ }^{8}$. Bacteroides vulgatus and Bacteroides thetaiotaomicron produce propionate through the succinate pathway. The main source of butyrate is in the clostridial clusters of obligate anaerobes, a small fraction of which produce propionate via the lactate pathway ${ }^{9}$. In addition to the SCFA regulation of intestinal homeostasis, gut-derived SCFAs can also affect parenteral tissues and organs through blood circulation ${ }^{10}$. SCFAs affect the immune response by regulating the activity of histone deacetylases in the immune cell genome and by regulating signalling through $\mathrm{G}$ protein-coupled receptors in immune cells ${ }^{11,12}$. In our previous study, we found a significant difference between the microbiome in patients with aplastic anaemia and that in normal controls. The abundances of Enterobacteriaceae and lactobacilli in the AA group were higher than those in the NC group, but the abundances of Bacteroides, Clostridium and Erysipelas were lower. At the species level, there are higher abundances of E. coli and Clostridium citroniae but lower abundances of Prevotella copri, Roseburia faecis, and Ruminococcus bromii ${ }^{7}$. Therefore, fatty acid levels in bone marrow supernatants and peripheral plasma were measured in patients with aplastic anaemia syndrome and normal controls. The differences in the levels of microorganisms, metabolic pathways and metabolites between the two groups were analysed by metagenomic sequencing. The aim of this study was to elucidate the changes in fatty acid metabolism in patients with aplastic anaemia and to determine how they might be related to gut microbes.

\section{Materials And Methods}

\section{Patients}

Twelve patients aged 15 to 81 years, with a median age of 46 years, were newly diagnosed with aplastic anaemia at the Tianjin Medical University General Hospital between September 2020 and June 2020 (AA group). Bone marrow supernatant, peripheral blood plasma and stool samples were collected before 
treatment. The admission criteria were as follows: patients who did not take antibiotics, probiotics or other haematologic diseases within 3 months prior to admission. Bone marrow supernatant, peripheral blood plasma and stool samples were also collected from ten healthy volunteers. This study was approved by the Ethics Committee of Tianjin Medical University General Hospital, and informed written consent was obtained from all patients or their parents according to the Declaration of Helsinki.

\section{Instruments and reagents}

The following instruments and reagents were used: a - $80^{\circ} \mathrm{C}$ refrigerator (Haier), a sterile EPPENDORF tube (Tianjin Haoyang Biotechnology Co., Ltd.), a Thermo Trace 1310-ISQ LT gas-mass spectrometer (Thermo), a refrigeration centrifuge (Hunan Instrument, H1850R), an electrothermal thermostatic water bath (Chang'an scientific instrument), a Covaris M220 ultrasound instrument (Covaris, Woburn, MA, USA), an NEBNext TM DNA Kit for Illumina (NEB, USA), a fluorescence quantizer (Qubit fluorometer, USA), and an Illumina NOVASE6000PE150. Phosphoric acid (Chinese medicine), ether (Chinese medicine), acetic acid (Sigma $\geq 99.5 \%$ ), propionic acid (Sigma $>99.0 \%$ ), butyric acid (Sigma $>99.0 \%$ ), isobutyric acid (Sigma $>99.0 \%$ ), valeric acid (Sigma $>98.0 \%$ ), isovaleric acid (Sigma > 99.0\%), hexanoic acid (Aladdin $\geq 99.5 \%$ ), isohexanoic acid (Sigma > 98\%), n-hexane (Chinese medicine), chloroform (Vokai), water (Millipore), sulfuric acid (Chinese medicine), methanol (Chinese medicine), methyl salicylate ( $\mathrm{TCl} \geq 99 \%), 52$ kinds of mixed fatty acid methyl esters, agarose (Chinese medicine). A QIAamp DNA Stool Mini Kit (Qiagen, Hilden, Germany), an Agencourt Ampure XP (Beckman, USA), and a GenNextTM NGS Library Quantification Kit.

\section{DNA extraction}

Total DNA was extracted from the faeces with a QIAamp DNA Stool Mini Kit (QIAGEN, Hilden, Germany). DNA completeness and purity were assessed by running the samples on $1.2 \%$ agarose gels. The concentration of DNA was determined on a Qubit fluorometer.

\section{Metagenomic library preparation and sequencing}

Extracted DNA was sheared with a Covaris M220 (Covaris, Woburn, MA, USA) programmed to generate 300-bp fragments. The sequencing libraries were constructed with a NEBNext ${ }^{\circledR}$ Ultra ${ }^{T M}$ DNA Library Prep Kit for Illumina ${ }^{\circledR}$ (NEB, USA). The products were purified using AgarosAgencourt AMPure XP (Beckman, USA) and quantified using a GenNextTM NGS Library Quantification Kit (Toyobo, Japan). The libraries were sequenced using Illumina NovaSeq 6000 and 150-bp paired-end technology at TinyGen Bio-Tech (Shanghai) Co., Ltd.

\section{Fatty acid sequencing}

The contents of long-chain fatty acids, including acetic acid, alanic acid, isobic acid, butyric acid, isoprene, ethionic acid, and hexacids, in the bone marrow of the AA patients and NCs were measured. The medium/long-chain fatty acids examined included the following: ingric acid, niacin, xian acid, lauric acid, thirteen alkyd, nutmeg acid, anti-9-nutmeg oleic acid, shun-9-nutmeg, xenoacid acid, anti-10-15 oleic acid, shun-10-ten pentaenoleic acid, palmitic acid, anti-9palm oleic acid, shun-9-palm oleic acid, hexane acid, anti-10-17oleic acid, shun-10-17oleic acid, stearic acid, trans rock celery acid, anti-oleic acid, anti-isoelic

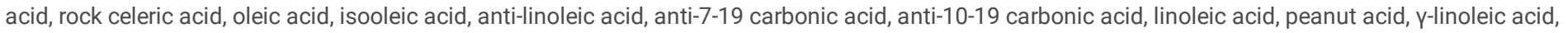
anti-11-20 carbonic acid, shun-20 11-20 carbonic acid, a-linoleic acid, 21 alkane acid, shun-11, 14-20 carbon dioleic acid, yamic acid, HOMO-y-linoleic acid, bajuic acid, mustard acid, shun-11, 14, 17-20 carbon trioleic acid, peanut tetraoleic acid, 23 alkane acid, shun-13, 16-22 carbon dioleic acid, EPA, wood wax acid, nerve acid, shun-7, 10, 13, 16-22 carbon tetraoleic acid, DPA, DPA, and DHA.

\section{Standard configuration}

The short-chain fatty acids examined were as follows: acetic acid, propylene acid, butyric acid, isobic acid, ethyl acid, isoprene, and hexacid. Pure standard products were mixed with ether to make nine standard concentration gradients $(0.02 \mathrm{sg} / \mathrm{mL}, 0.1 \mathrm{sg} / \mathrm{mL}, 1 \mathrm{sg} / \mathrm{mL}, 2 \mathrm{sg} / \mathrm{mL}, 5 \mathrm{sg} / \mathrm{mL} \otimes 10 \mu \mathrm{g} / \mathrm{mL} \otimes 25 \mu \mathrm{g} / \mathrm{mL} \bigotimes$ $50 \mu \mathrm{g} / \mathrm{mL} \rrbracket$ and $100 \mu \mathrm{g} / \mathrm{mL}$ ). Both the stock solution and the standard solution were stored at $0{ }^{\circ} \mathrm{C}$.

The medium/long chain fatty acids were synthesized as follows: 52 fatty acid methyl ester solutions were mixed with positive hexane to make 10 standard concentration gradients ( $1 \mathrm{sg} / \mathrm{mL}, 5 \mathrm{sg} / \mathrm{mL}, 10 \mathrm{sg} / \mathrm{mL}, 25 \mathrm{sg} / \mathrm{mL}, 50 \mathrm{sg} / \mathrm{mL}, 100 \mu \mathrm{g} / \mathrm{mL}, 250 \mu \mathrm{g} / \mathrm{mL}, 500 \mu \mathrm{g} / \mathrm{mL}, 1000 \mu \mathrm{g} / \mathrm{mL}, \mathrm{and} 2000 \mu \mathrm{g} / \mathrm{mL}$ ). The concentration is the total concentration of each component ( 51 standard fatty acid methyl esters). The stock solution was stored at $-80{ }^{\circ} \mathrm{C}$ and is now available as a standard solution.

\section{Sample pretreatment}

The short-chain fatty acids were synthesized as follows: the sample was transferred to a $2 \mathrm{~mL}$ centrifuge tube; $50 \mu \mathrm{L}$ of $15 \%$ phosphoric acid, $10 \mu \mathrm{L}$ of 75 $\mu \mathrm{g} / \mathrm{mL}$ of the internal label (iso-acid) solution and $140 \mu \mathrm{L}$ of ether were added; the mixture was subjected to vortex oscillation for $1 \mathrm{~min}$ and centrifugation for $10 \mathrm{~min}$ at $4{ }^{\circ} \mathrm{C}(12000 \mathrm{rpm})$ for liquidation detection.

The medium/long-chain fatty acids were synthesized as follows: the appropriate amount of sample was transferred to a $15 \mathrm{~mL}$ centrifuge tube; $2 \mathrm{~mL} 1 \%$ methanol sulfate solution was added and mixed for $1 \mathrm{~min}$; the mixture was esterified for $30 \mathrm{~min}$ in a water bath at $80^{\circ} \mathrm{C}$; the solution was removed and cooled; $1 \mathrm{~mL}$ positive hexane extraction was added, the mixture was washed with $5 \mathrm{~mL} \mathrm{H}_{2} \mathrm{O}\left(4^{\circ} \mathrm{C}\right)$, centrifuged at $12000 \mathrm{rpm}$ at $4{ }^{\circ} \mathrm{C}$ for 10 min, placed in an oscillating mixer for $30 \mathrm{~s}$, centrifuged at $12000 \mathrm{rpm}$ for $5 \mathrm{~min}$, and subjected to precise absorption of $300 \mathrm{sL}$ of upper liquid in a $2 \mathrm{~mL}$ centrifuge tube; $15 \mathrm{sl}$ $500 \mathrm{ppm}$ salicylic acid was added as the internal label; and the mixture was placed in an oscillating mixer for 10 sand subjected to precise absorption of 250 $\mathrm{sL}$ (added to the test bottle). 


\section{GC-MS detection}

The chromatographic conditions were as follows: an Agilent HP-INNOWAX capillary column (30 $\mathrm{m} \times 0.25 \mathrm{~mm}$ ID $\times 0.25 \mu \mathrm{m})$ was used; the inlet temperature was $250^{\circ} \mathrm{C}$, the ion source temperature was $230^{\circ} \mathrm{C}$, and the transmission line temperature was $250{ }^{\circ} \mathrm{C}$ for the four poles; the bar temperature was $150{ }^{\circ} \mathrm{C}$. The program began at a starting temperature of $90^{\circ} \mathrm{C}$, was increased to $120^{\circ} \mathrm{C}$, was increased to $150^{\circ} \mathrm{C}$ at a rate of $5^{\circ} \mathrm{C} / \mathrm{min}$, and then was increased to $250^{\circ} \mathrm{C}$ at a rate of $25^{\circ} \mathrm{C} / \mathrm{min}$. The helium carrier had a carrier flow rate of $1.0 \mathrm{~mL} / \mathrm{min}$. MS conditions were as follows: electron blast ionization (El) source, SIM scan mode, and electron energy: $70 \mathrm{eV}$.

\section{Data analysis:}

The raw fastq files were demultiplexed based on the index. The raw, paired-end reads were trimmed and quality controlled using Trimmomatic (http://www.usadellab.org/cms/?page=trimmomatic) and cutadapt software. The host sequences were removed by using BWA (http://biobwa.sourceforge.net/). The reformatted data were assembled and predicted by Megahit (https://github.com/voutcn/megahit) and METAProdigal (http://prodigal.ornl.gov/) software. The predicted genes were clustered, and a nonredundant gene catalogue was constructed with the CD-HIT package (http://www.bioinformatics.org/cd-hit/) (parameters: identity=95\%, coverage=90\%). The abundance of genes was quickly estimated by genomeCoverageBed in bedtools. The representative sequences were assigned for taxonomy against the NR database with an e-value of 1e-5 by DIAMOND software. The taxonomic information included the domain, kingdom, phylum, class, order, family, genus, and species. To understand the functions of the differentially expressed genes, the genes were assigned against the eggNOG database, KEGG database, GO database, CAZy database and ARDB database by using BLASTP (BLAST Version 2.2.28+, http://blast.ncbi.nlm.nih.gov/Blast.cgi) with an e-value of 1e-5.

For the bar graphs, VENN, heatmap analysis, PCA, PCoA, and NMDS were used, and the results were plotted with R (Version 3.4.1). Corr. R language (psych package) was used to determine the abundances of the differential expressed fatty acids and genes. Spearman correlation analysis was carried out by the test function, and visualization was carried out by the pheatmap package.

\section{Results}

\section{Bone marrow fatty acid metabolism in patients with aplastic anaemia:}

\section{Changes in supernatant fatty acids in the bone marrow of patients with aplastic anaemia:}

Orthogonal least squares discriminant analysis (OPLS-DA) were used to analyse the major fatty acids in the AA and NC groups (Tab 1,Fig 1). In the bone marrow supernatant, there was a significant difference in the short-chain fatty acid compositions, while there were no significant differences in the mediumand long-chain fatty acid compositions. There were significant differences in 3 of the 7 short-chain fatty acids tested. Among the 52 types of medium- and long-chain fatty acids detected, only one showed a significant difference. The major difference between patients with aplastic anaemia and normal controls was short-chain fatty acid metabolism.

Tab 1:OPLS-DA analysis

\begin{tabular}{lllll} 
& pre & R2X(cum) & R2Y(cum) & Q2(cum) \\
\hline AABM vsNCBM $\triangle$ C6-C24FA & 2 & 0.543 & 0.614 & 0.272 \\
\hline AABM vs NCBM $\triangle$ C1-C6FA囚 & $1+4+0$ & 0.965 & 0.881 & 0.732
\end{tabular}

Compared to the NC group, the contents of docosatetraenoate $(C 22: 4)(p<0.05)$ were significantly decreased, and the contents of isovaleric acid ( $p<0.001)$, isobutyric acid $(p<0.0001)$ and valeric acid $(p<0.005)$ were significantly increased (Tab 2, Fig 2$)$.

Tab 2 .Changed fatty acids in the bone marrow

\begin{tabular}{llllllll} 
& mean_AABM & sd_AABM & mean_NCBM & sd_NCBM & Fold Change_AABM/NCBM & log2(FC_AABM/NCBM) & p.value \\
\hline C22:4 & 3.290286 & 0.954465 & 4.503714 & 1.01609 & 0.73057 & -0.4529 & 0.011129 \\
\hline Isobutyric acid & 0.03425 & 0.014858 & 0.0124 & 0.005125 & 2.7621 & 1.4658 & $9.79 \mathrm{E}-05$ \\
\hline Isovaleric acid & 0.030167 & 0.01777 & 0.0159 & 0.002726 & 1.8973 & 0.92393 & 0.000159 \\
\hline Valeric acid & 0.025583 & 0.011033 & 0.0141 & 0.002767 & 1.8144 & 0.85951 & 0.002924 \\
\hline Acetic acid & 2.572667 & 0.974088 & 1.5007 & 0.520259 & 1.7143 & 0.77763 & 0.005073
\end{tabular}

Changes in plasma fatty acids in patients with aplastic anaemia:

OPLS-DA were used to analyse the major fatty acids in the AA and NC groups (Tab 3 and Figs 3). In the peripheral plasma, there were significant differences in medium- and long-chain fatty acid compositions, while there were no significant differences in short-chain fatty acid copositions. Among the 52 types of

Page $4 / 38$ 
medium- and long-chain fatty acids detected, there were significant differences in the contents of 16 . Only one of the seven short-chain fatty acids evaluated showed a significant difference. The major difference between aplastic anaemia patients and normal controls is short-chain fatty acid metabolism.

Tab 3 the OPLS-DA analysis of fatty acids in plasma

$\begin{array}{lllll} & \text { pre } & \text { R2X(cum) } & \text { R2Y(cum) } & \text { Q2(cum) } \\ \text { AAPB vs NCPB囚C6-C24FA』 } & 3 & 0.74 & 0.837 & 0.535 \\ \text { AAPB vs NCPB囚C1-C6FA』 } & 1+2+0 & 0.935 & 0.357 & 0.276\end{array}$

Compared to the NC group, in the AA group, the contents of myristelaidate $(C 14: 1 T)(p<0.001)$ and isobutyric acid $(p<0.05)$ were increased significantly.

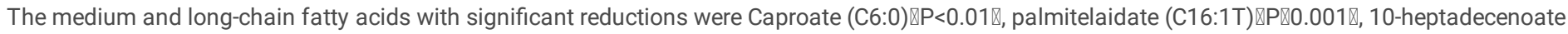
$(C 17: 1) \otimes P<0.01 \rrbracket, 10$-transsheptadecenoate $(C 17: 1 T) \otimes P<0.05 \rrbracket$, stearate $(C 18: 0) \otimes P<0.01 \rrbracket$, petroselaidate $(C 18: 1 N 12 T) \otimes P<0.01 \rrbracket$, transvaccenate $(C 18: 2 N 7 T)$ $\triangle P<0.05 \rrbracket$, linoelaidate $(C 18: 2 N 6 T) \otimes P<0.05 \rrbracket$, 7-transnonadecenoate $(C 19: 1 N 12 T) \otimes P<0.001 \rrbracket$, eicosenoate $(C 20: 1) \otimes P<0.05 \rrbracket$, trans 11-eicosenoate $(C 20: 1 T)$ $\varangle \mathrm{P}<0.01 \rrbracket$, erucate $(\mathrm{C} 22: 1 \mathrm{~N} 9) \varangle \mathrm{P}<0.05 \rrbracket$, brassidate $(\mathrm{C} 22: 1 \mathrm{~N} 9 \mathrm{~T}) \otimes \mathrm{P}<0.05 \rrbracket$, docosadienoate $(\mathrm{C} 22: 2) \otimes \mathrm{P}<0.05 \rrbracket$, docosatetraenoate $(\mathrm{C} 22: 4) \otimes \mathrm{P}<0.05 \rrbracket$, and

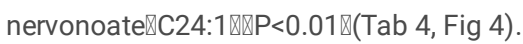

Tab 4 changed fatty acids(including long-chain fatty acids and short-chain fatty acids) in AA group in plasm.

\begin{tabular}{|c|c|c|c|c|c|c|c|}
\hline & mean_AAB & sd_AAB & mean_NCB & sd_NCB & Fold Change_AAB/NCB & $\log 2\left(\mathrm{FC} \_\mathrm{AAB} / \mathrm{NCB}\right)$ & p.value \\
\hline C14:1T & 20.41007 & 6.087152 & 14.15148 & 2.00612 & 1.4423 & 0.52833 & 0.000869 \\
\hline C19:1N12T & 4.507297 & 0.7233 & 6.090836 & 0.770336 & 0.74001 & -0.43438 & 0.000869 \\
\hline C16:1T & 2.730239 & 0.552947 & 3.7321 & 0.519632 & 0.73156 & -0.45096 & 0.001736 \\
\hline C17:1 & 3.526343 & 0.774811 & 4.735835 & 1.068266 & 0.74461 & -0.42545 & 0.006211 \\
\hline $\mathrm{C} 24: 1$ & 3.344069 & 0.897316 & 4.843347 & 1.091746 & 0.69045 & -0.5344 & 0.006211 \\
\hline C18:0 & 405.9224 & 57.07996 & 481.828 & 62.47371 & 0.84246 & -0.24731 & 0.009199 \\
\hline C18:1N12T & 1.851153 & 0.43985 & 2.313218 & 0.34065 & 0.80025 & -0.32148 & 0.009199 \\
\hline C20:1T & 3.401346 & 0.501943 & 4.125306 & 0.597002 & 0.82451 & -0.2784 & 0.009199 \\
\hline C17:1T & 4.212472 & 0.721826 & 5.238189 & 0.767364 & 0.80418 & -0.3144 & 0.011129 \\
\hline C20:1 & 4.555985 & 0.75348 & 5.501479 & 0.752388 & 0.82814 & -0.27206 & 0.011129 \\
\hline C6:0 & 0.441762 & 0.28031 & 0.615143 & 0.140688 & 0.71814 & -0.47765 & 0.013306 \\
\hline C22:1N9T & 3.958385 & 0.607051 & 4.667704 & 0.664834 & 0.84804 & -0.2378 & 0.022914 \\
\hline $\mathrm{C} 22: 1 \mathrm{~N} 9$ & 7.894534 & 1.210693 & 9.309187 & 1.325933 & 0.84804 & -0.2378 & 0.022914 \\
\hline $\mathrm{C} 22: 2$ & 0.969069 & 0.331208 & 1.325357 & 0.314959 & 0.73118 & -0.45171 & 0.022914 \\
\hline C22:4 & 2.972657 & 1.104194 & 3.86212 & 1.149583 & 0.7697 & -0.37764 & 0.022914 \\
\hline C18:2N6T & 1.0497 & 0.258317 & 1.28606 & 0.226167 & 0.81621 & -0.29298 & 0.02718 \\
\hline C18:1N7T & 7.464862 & 1.388793 & 8.935318 & 1.527666 & 0.83543 & -0.2594 & 0.037797 \\
\hline Isobutyric acid & 0.039 & 0.053662 & 0.0136 & 0.01152 & 2.8676 & 1.5199 & 0.034652 \\
\hline
\end{tabular}

\section{Faecal microbe changes in patients with aplastic anaemia:}

Metagenomic sequencing showed that the microbial abundance in the faecal microflora of the AA group was changed at six levels: phylum, class, order, family, genus and species.

\section{Species abundance changes in the AA group}

$\beta$ diversity analysis showed no significant difference in microbial abundance between the AA and NC groups, but the tendency of grouping is obvious.(Fig 5).

Phylum level: According to the a diversity analysis, there was no difference in coverage, sobs, or the Chao, ACE or Simpson index between the AA and NC groups, but the difference in the Shannon index, which was a parameter of colony diversity, was significant $(p=0.010)($ Fig 6$)$. The analysis of group differences confirmed that the overall microbial abundances of bacteria and eukaryotes at the phylum level were decreased in the AA group. The microbial abundances of Candidatus__ Zambryskibacteria, Candidatus_ Falkowbacteria, Candidatus_ Yanofskybacteria and Nematoda were increased in the AA group (Tab 5, Fig 7). 
Tab 5. .the specific changed ID in the phylum level

\begin{tabular}{lllllllll} 
level & .ID & p.value & p.signif & method & mean(AA) & n(AA) & mean(NC) & n(NC) \\
\hline B & p_ & 0.005645 & $* *$ & Wilcoxon & 5326.235 & 12 & 8399.529 & 10 \\
\hline B & p_Candidatus_Falkowbacteria & 0.043118 & $*$ & Wilcoxon & 8.560287 & 12 & 1.824681 & 10 \\
\hline B & p_Candidatus_Yanofskybacteria & 0.035419 & $*$ & Wilcoxon & 0.015688 & 12 & 9.207157 & 10 \\
\hline B & p_Candidatus_Zambryskibacteria & 0.019208 & $*$ & Wilcoxon & 0.418214 & 12 & 0.118341 & 10 \\
\hline E & P_ & 0.011153 & $*$ & Wilcoxon & 7.880836 & 12 & 1275.989 & 10 \\
\hline E & P_Nematoda & 0.04257 & $*$ & Wilcoxon & 223.77 & 12 & 327.3807 & 10
\end{tabular}

Class level $\mathbb{\text { In }}$ the AA group, there were no significant differences in a diversity (Fig 8). The results showed no significant changes in the sequencing depth, species quantity, distribution uniformity, colony abundance, diversity, or microbial diversity in the AA group. The differential analysis showed that the abundances of Sphingobacteriia (bacteria), Oomycetes and Enoplea (eukaryotes) were decreased in the AA group (Tab 6, Fig 9).

Tab 6.the specific changed ID in the class level

\begin{tabular}{lllllllll} 
level & .ID & p.value & p.signif & method & mean(AA) & n(AA) & mean(NC) & n(NC) \\
\hline B & C_Sphingobacteriia & 0.029961 & $*$ & Wilcoxon & 56.00991 & 12 & 140.242 & 10 \\
\hline E & C_Oomycetes & 0.008957 & $* *$ & Wilcoxon & 0.659821 & 12 & 6.989042 & 10 \\
\hline E & C_Enoplea & 0.04257 & $*$ & Wilcoxon & 217.3663 & 12 & 326.564 & 10
\end{tabular}

Order level: The same a diversity analysis showed no significant difference between the two groups(Fig 10). Specific difference analysis revealed decreased abundances of the bacterial orders Burkholderiales, Enterobacterales and Sphingobacteriales and the eukaryotic orders Peronosporales and Trichinellida in the AA group (Tab 7, Fig 11).

Tab 7. the specific changed ID in the order level

\begin{tabular}{lllllllll} 
level & .ID & p.value & p.signif & method & mean(AA) & n(AA) & mean(NC) & n(NC) \\
\hline B & O_Burkholderiales & 0.029961 & $*$ & Wilcoxon & 798.2971 & 12 & 3142.975 & 10 \\
\hline B & O_Rhodobacterales & 0.035828 & $*$ & Wilcoxon & 23.62861 & 12 & 43.59014 & 10 \\
\hline B & O_Sphingobacteriales & 0.029961 & $*$ & Wilcoxon & 55.21925 & 12 & 140.2076 & 10 \\
\hline E & O_Peronosporales & 0.008957 & $* *$ & Wilcoxon & 0.659336 & 12 & 6.850161 & 10 \\
\hline E & O_Trichinellida & 0.04257 & $*$ & Wilcoxon & 217.3663 & 12 & 326.564 & 10
\end{tabular}

Family level: At the family level, the a diversity analysis also showed no significant difference between the two (Fig 12). The microbial abundances of Peronosporaceae, the Trichuridae family, alcaligenaceae in the eukaryotic kingdom, Rhodobacteraceae, Ruminococcaceae, Sphingobacteriaceae and Yersiniaceae family in the bacterial kingdom were decreased in the AA group. The microbial abundance of the Enterocytozoonidae family of the eukaryotic kingdom was elevated in the AA group (Tab 8, Fig 13).

Tab 8. the specific changed ID in the family level 


\begin{tabular}{lllllllll} 
level & .ID & p.value & p.signif & method & mean(AA) & n(AA) & mean(NC) & n(NC) \\
\hline B & f_Alcaligenaceae & 0.0169 & $*$ & Wilcoxon & 15.96707 & 12 & 22.477 & 10 \\
\hline B & f_Rhodobacteraceae & 0.035828 & $*$ & Wilcoxon & 23.18635 & 12 & 42.36301 & 10 \\
\hline B & f_Ruminococcaceae & 0.02058 & $*$ & Wilcoxon & 113635.9 & 12 & 47871.68 & 10 \\
\hline B & f_Sphingobacteriaceae & 0.02058 & $*$ & Wilcoxon & 53.83421 & 12 & 131.7942 & 10 \\
\hline B & f_YYersiniaceae & 0.024916 & $*$ & Wilcoxon & 120.5516 & 12 & 153.3019 & 10 \\
\hline E & f_Peronosporaceae & 0.008957 & $* *$ & Wilcoxon & 0.659336 & 12 & 6.850161 & 10 \\
\hline E & f_Enterocytozoonidae & 0.015062 & $*$ & Wilcoxon & 0.964247 & 12 & 0.124079 & 10 \\
\hline E & f_Trichuridae & 0.04257 & $*$ & Wilcoxon & 217.3663 & 12 & 326.564 & 10
\end{tabular}

Genus level: There was no significant difference in a diversity at the genus level(Fig 14). In eukaryotes, however, the abundances of Arabidopsis, Cucurbita and Enterocytozoon, as well as Cruoricaptor, Absiella, Afifella, Desulfobacca, Desulfosarcina, Dokdonia, Drancourtella, Enhydrobacter, Helicobacter, Lentzea, Rhodoferax, Smithella, Subdoligranulum, and Thiomargarita in the bacterial kingdom, were increased in the AA group. The abundances of Lambdavirus, Rtpvirus, Achromobacter, Burkholderia, Mycoavidus, Thiomonas, Caecibacter, Catenibacterium, Chloroherpeton, Citrobacter, Desulfatirhabdium, Friedmanniella, Haematomicrobium, Megasphaera, Oceanicaulis, Petrimonas, Serratia, Sneathia, Treptobacillus, Succinispira, and Sulfuricurvum in the bacterial kingdom showed the opposite trend (Tab 9, Fig 15).

Tab 9. the specific changed ID in the genus level 


\begin{tabular}{|c|c|c|c|c|c|c|c|c|}
\hline level &.$I D$ & p.value & p.signif & method & mean(AA) & $n(A A)$ & mean(NC) & $\mathrm{n}(\mathrm{NC})$ \\
\hline B & g_Achromobacter & 0.007145 & $\star \star$ & Wilcoxon & 10.09373 & 12 & 12.26749 & 10 \\
\hline B & g_Burkholderia & 0.003436 & $\star *$ & Wilcoxon & 14.80387 & 12 & 31.55629 & 10 \\
\hline B & g_Cruoricaptor & 0.008635 & ** & Wilcoxon & 103.0674 & 12 & 0.00011 & 10 \\
\hline B & g_Mycoavidus & 0.005645 & ** & Wilcoxon & 0.850142 & 12 & 5.981828 & 10 \\
\hline B & g_Thiomonas & 0.008957 & $\star \star$ & Wilcoxon & 8.839875 & 12 & 26.5866 & 10 \\
\hline B & g_Absiella & 0.029961 & * & Wilcoxon & 205.4508 & 12 & 89.55985 & 10 \\
\hline B & g_Afifella & 0.027812 & * & Wilcoxon & 0.100013 & 12 & 0 & 10 \\
\hline B & g_Caecibacter & 0.015976 & * & Wilcoxon & 0.729265 & 12 & 15.54945 & 10 \\
\hline B & g_Catenibacterium & 0.02058 & * & Wilcoxon & 305.7946 & 12 & 2616.767 & 10 \\
\hline B & g_Chloroherpeton & 0.028942 & * & Wilcoxon & 0.004109 & 12 & 0.128666 & 10 \\
\hline B & g_Citrobacter & 0.04257 & * & Wilcoxon & 736.4254 & 12 & 2760.517 & 10 \\
\hline B & g_Desulfatirhabdium & 0.02215 & * & Wilcoxon & 0.157288 & 12 & 0.743299 & 10 \\
\hline B & g_Desulfobacca & 0.013201 & * & Wilcoxon & 0.065557 & 12 & 0 & 10 \\
\hline B & g_Desulfosarcina & 0.029085 & * & Wilcoxon & 0.209741 & 12 & 0.168518 & 10 \\
\hline B & g_Dokdonia & 0.035916 & * & Wilcoxon & 0.552809 & 12 & 0.037748 & 10 \\
\hline B & g_Drancourtella & 0.024916 & * & Wilcoxon & 499.5553 & 12 & 131.7744 & 10 \\
\hline B & g_Enhydrobacter & 0.018256 & * & Wilcoxon & 0.629597 & 12 & 0.045162 & 10 \\
\hline B & g_Friedmanniella & 0.035706 & * & Wilcoxon & 0.019968 & 12 & 0.512966 & 10 \\
\hline B & g_Haematomicrobium & 0.018657 & * & Wilcoxon & 0.010867 & 12 & 0.541071 & 10 \\
\hline B & g_Helicobacter & 0.04257 & * & Wilcoxon & 119.9702 & 12 & 50.31599 & 10 \\
\hline B & g_Lentzea & 0.029085 & * & Wilcoxon & 0.151672 & 12 & 0.007282 & 10 \\
\hline B & g_Megasphaera & 0.0169 & * & Wilcoxon & 120.5764 & 12 & 5919.09 & 10 \\
\hline B & g_Oceanicaulis & 0.021299 & * & Wilcoxon & 0 & 12 & 1.017431 & 10 \\
\hline B & g_Petrimonas & 0.032066 & * & Wilcoxon & 1.570891 & 12 & 15.53802 & 10 \\
\hline B & g_Rhodoferax & 0.042226 & * & Wilcoxon & 0.437173 & 12 & 0.068321 & 10 \\
\hline B & g_Serratia & 0.0169 & * & Wilcoxon & 93.35629 & 12 & 116.9199 & 10 \\
\hline B & g_Smithella & 0.035001 & * & Wilcoxon & 1.334781 & 12 & 0.008958 & 10 \\
\hline B & g_Sneathia & 0.026317 & * & Wilcoxon & 0.0823 & 12 & 2.193245 & 10 \\
\hline B & g_Streptobacillus & 0.035828 & * & Wilcoxon & 4.371066 & 12 & 9.960238 & 10 \\
\hline B & g_Subdoligranulum & 0.035828 & * & Wilcoxon & 6958.998 & 12 & 2711.458 & 10 \\
\hline B & g_Succinispira & 0.011106 & * & Wilcoxon & 0.329153 & 12 & 1.923889 & 10 \\
\hline B & g_Sulfuricurvum & 0.024787 & * & Wilcoxon & 0.163208 & 12 & 0.507412 & 10 \\
\hline B & g_Thiomargarita & 0.01856 & * & Wilcoxon & 5.323346 & 12 & 0.009884 & 10 \\
\hline B & s_Armatimonadetes_bacterium_CG_4_8_14_3_um_filter_66_20 & 0.027812 & * & Wilcoxon & 0.068908 & 12 & 0 & 10 \\
\hline $\mathrm{E}$ & g_Arabidopsis & 0.034593 & * & Wilcoxon & 0.131755 & 12 & 0.006128 & 10 \\
\hline $\mathrm{E}$ & g_Cucurbita & 0.027812 & * & Wilcoxon & 0.037481 & 12 & 0 & 10 \\
\hline$E$ & g_Enterocytozoon & 0.015062 & * & Wilcoxon & 0.964247 & 12 & 0.124079 & 10 \\
\hline$E$ & g_Plasmopara & 0.01377 & * & Wilcoxon & 0.629125 & 12 & 6.22372 & 10 \\
\hline$E$ & g_Trichuris & 0.04257 & * & Wilcoxon & 217.3663 & 12 & 326.564 & 10 \\
\hline V & g_Lambdavirus & 0.006674 & ** & Wilcoxon & 0.13463 & 12 & 2.01234 & 10 \\
\hline v & g_Rtpvirus & 0.008557 & ** & Wilcoxon & 0.001132 & 12 & 912.9737 & 10 \\
\hline
\end{tabular}


Species level: Similarly, there were no significant differences observed in the a diversity analysis at the species level(Fig 16). However, in the AA group, the abundances of 84 species were increased, including 2 species in the viral kingdom (Staphylococcus_phage_phiSA_BS2, Streptococcus_phage_P0092), 2 species in the eukaryotic kingdom (Candida_maltosa, Enterocytozoon_bieneusi), 2 species in the archaea kingdom (Methanobacterium_congolense, Methanosarcina_spelaei) and 78 species in the bacterial kingdom (Proteus species, Clostridioides species, Ruminococcus species, etc.). A total of 125 microbial species were downregulated in the AA group, including 6 species in the viral kingdom $₫$ Stx 1 -converting_phage_phi-0153 ,

Enterococcus_phage_EFDG1 ,Enterococcus_phage_EFLK1 , Escherichia_phage_vB_Ecos_CEB_EC3a, Escherichia_virus_186, Streptococcus_virus_ALQ132】, 1 species in the eukaryotic kingdom (Plasmopara_halstedi) $₫ 2$ species in the archaea kingdom $₫$ Candidatus_Altiarchaeales_archaeon_WOR_SM1_79,

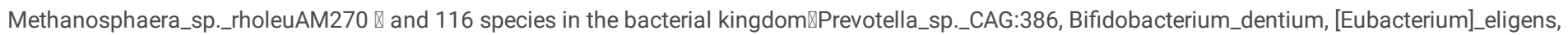
Clostridium_sp._CAG:7, Catenibacterium_mitsuokai, etc(Tab 10,Fig 17).

Tab 10. the specific changed IDs in the species level(only shows the top 20 species ID)

\begin{tabular}{|c|c|c|c|c|c|c|c|}
\hline. ID & p.value & p.signif & method & mean(AA) & $\mathrm{n}(\mathrm{AA})$ & mean(NC) & $n(N C)$ \\
\hline s_Subdoligranulum_sp._APC924/74 & 0.035828 & * & Wilcoxon & 4554.301 & 12 & 611.9317 & 10 \\
\hline s_Erysipelotrichaceae_bacterium_3_1_53 & 0.0169 & * & Wilcoxon & 3758.78 & 12 & 76.15913 & 10 \\
\hline s_Ruminococcus_sp. & 0.04257 & * & Wilcoxon & 2141.948 & 12 & 97.13947 & 10 \\
\hline s_Bifidobacterium_dentium & 0.04257 & * & Wilcoxon & 729.3444 & 12 & 6287.88 & 10 \\
\hline S_Absiella_dolichum & 0.029961 & * & Wilcoxon & 205.4508 & 12 & 89.55985 & 10 \\
\hline s_Drancourtella_massiliensis & 0.029961 & * & Wilcoxon & 165.5897 & 12 & 11.95476 & 10 \\
\hline S_Clostridiales_bacterium_VE202-13 & 0.029961 & * & Wilcoxon & 160.2015 & 12 & 31.45474 & 10 \\
\hline s_Clostridium_acetireducens & 0.04257 & * & Wilcoxon & 86.51676 & 12 & 38.70515 & 10 \\
\hline s_Fusobacterium_sp._CM21 & 0.02058 & * & Wilcoxon & 68.64265 & 12 & 25.16005 & 10 \\
\hline s_Bifidobacterium_saeculare & 0.02058 & * & Wilcoxon & 65.66262 & 12 & 16.88376 & 10 \\
\hline s_Curvibacter_putative_symbiont_of_Hydra_magnipapillata & 0.035828 & * & Wilcoxon & 57.01436 & 12 & 9.584941 & 10 \\
\hline s_uncultured_actinobacterium_HF0500_35G12 & 0.003436 & ** & Wilcoxon & 14.16386 & 12 & 2.742277 & 10 \\
\hline s_Citrobacter_werkmanii & 0.024916 & * & Wilcoxon & 14.11588 & 12 & 118.4372 & 10 \\
\hline s_Enterococcus_saccharolyticus & 0.037581 & * & Wilcoxon & 11.35573 & 12 & 0.137005 & 10 \\
\hline
\end{tabular}

\section{Changes in the KEGG signalling pathway in the AA group}

Compared with the normal control group, there were significant differences in the abundances of genes involved in 12 signalling pathways in the AA group. Among them, the abundances of genes involved in 2 metabolism-related pathways were upregulated (penicillin and cephalosporin biosynthesis and tyrosine and tryptophan biosynthesis). In addition, the abundances of genes involved in eight metabolism-related pathways, including nonribosomal peptides, betalain biosynthesis, and lysine degradation, showed the opposite trend. We also observed the downregulation of genes involved in pathways related to type I diabetes mellitus in humans (Fig 18, Tab 11).

Tab 11.Changed abundance of specific KEGG pathway ID in AA group 


\begin{tabular}{|c|c|c|c|c|c|c|c|c|}
\hline ID & p.value & p.format & p.signif & method & mean(AA) & $\mathrm{n}(\mathrm{AA})$ & mean(NC) & $\mathrm{n}(\mathrm{NC})$ \\
\hline Betalain biosynthesis & 0.035828 & 0.036 & * & Wilcoxon & 23.44387 & 12 & 30.77787 & 10 \\
\hline Biosynthesis of siderophore group nonribosomal peptides & 0.024916 & 0.025 & * & Wilcoxon & 202.6813 & 12 & 394.1924 & 10 \\
\hline Caprolactam degradation & 0.035828 & 0.036 & * & Wilcoxon & 61.81826 & 12 & 90.93364 & 10 \\
\hline Drug metabolism - cytochrome P450 & 0.04257 & 0.043 & * & Wilcoxon & 148.2111 & 12 & 213.5611 & 10 \\
\hline Lysine degradation & 0.029961 & 0.030 & * & Wilcoxon & 701.4266 & 12 & 923.5934 & 10 \\
\hline Metabolism of xenobiotics by cytochrome P450 & 0.04257 & 0.043 & * & Wilcoxon & 148.3814 & 12 & 215.1718 & 10 \\
\hline N-Glycan biosynthesis & 0.035828 & 0.036 & * & Wilcoxon & 63.28539 & 12 & 95.42845 & 10 \\
\hline Penicillin and cephalosporin biosynthesis & 0.0169 & 0.017 & * & Wilcoxon & 197.2371 & 12 & 136.6711 & 10 \\
\hline Phenylalanine, tyrosine and tryptophan biosynthesis & 0.04257 & 0.043 & * & Wilcoxon & 4709.729 & 12 & 4261.92 & 10 \\
\hline Type I diabetes mellitus & 0.029961 & 0.030 & * & Wilcoxon & 371.8122 & 12 & 426.242 & 10 \\
\hline Ubiquinone and other terpenoid-quinone biosynthesis & 0.035828 & 0.036 & * & Wilcoxon & 978.2562 & 12 & 1304.532 & 10 \\
\hline Valine, leucine and isoleucine degradation & 0.011153 & 0.011 & * & Wilcoxon & 1148.773 & 12 & 1354.179 & 10 \\
\hline
\end{tabular}

\section{Changes in fatty acid levels in the AA group were associated with microbial species}

\section{Correlation between fatty acid content in the bone marrow supernatant and faecal microbial abundance}

The abundance of the uncultured_actinobacterium_ HF0500_35g12 species in the bacterial kingdom was positively correlated with the valeric acid content, which is negatively correlated with the microbial abundances of the Candidatus__ Zambryskibacteria phylum, the genus Cruoricaptor, the Cruoricaptor_ ignavus species, the genus drancourtella, the Bifidobacterium_Saeculare species, the Drancourtella_ Massiliensis species, the Pseudomonas_Syringae species, the Sulfurospirillum_ Halorespirans species and the Staphylococcus_phage_phiSA_BS2 species in the viral kingdom. There as a positive correlation between isobutyric acid and the microbial abundances of the Sphingobacteriia class, Sphingobacteriales order, Sphingobacteriaceae family, Afifella genus , and Enterococcus_sp._4E1_DIV0656 species. The abundance of microbes in the bacterial kingdom that showed a negative correlation with the isobutyrate content included those in the Absiella genus, Absiella_dolichum species, Cruoricaptor genus, Cruoricaptor_ignavus species, Mycoavidus genus , Mycoavidus_cysteinexigens species and 8 other microbial species. The abundance of Catenibacterium genus and Catenibacterium_mitsuokai speices in the bacterial kingdom was positively correlated with the content of Docosatetraenoate, which is negatively correlated with the microbial abundance of the Enhydrobacter genus, Enhydrobacter_aerosaccus species, Smithella genus, Bifidobacterium_sp._MSTE12 genus and 7 other species. The content of isovaleric acid showed a negative correlation with the abundances of the Drancourtella genus, Chloroflexi_bacterium_GWB2_49_20 species,

Geobacillus_thermodenitrificans species, Kocuria_kristinae species, and Polaromonas_sp._JS666 species in the bacterial kingdom and the Methanobacterium_congolense species in archaea kingdom (Tab 12, Fig 19).

Tab 12.the specific correlation between the microbial abundance and fatty acids in the bone marrow supernatant in AA group(the species level showed in supplementary files) 


\begin{tabular}{lllllll} 
nameA & nameB & correlation & P.value & method & star & group \\
\hline B_c_Sphingobacteriia & Isobutyric.acid & 0.677197 & 0.015554 & spearman & * & AA \\
\hline B_f_Sphingobacteriaceae & Isobutyric.acid & 0.722811 & 0.007911 & spearman & ** & AA \\
\hline B_g_Absiella & Isobutyric.acid & -0.61404 & 0.033666 & spearman & * & AA \\
\hline B_g_Afifella & Isobutyric.acid & 0.626033 & 0.029428 & spearman * & AA \\
\hline B_g_Cruoricaptor & Isobutyric.acid & -0.63042 & 0.027977 & spearman * & AA \\
\hline B_g_Mycoavidus & Isobutyric.acid & -0.62106 & 0.031136 & spearman * & AA \\
\hline B_o_Sphingobacteriales & Isobutyric.acid & 0.722811 & 0.007911 & spearman & $* *$ & AA \\
\hline B_g_Drancourtella & Isovaleric.acid & -0.61378 & 0.033762 & spearman & $*$ & AA \\
\hline B_g_Cruoricaptor & Valeric.acid & -0.65606 & 0.020517 & spearman & * & AA \\
\hline B_g_Drancourtella & Valeric.acid & -0.68652 & 0.013674 & spearman & * AA \\
\hline B_p_Candidatus_Zambryskibacteria & Valeric.acid & -0.60596 & 0.036759 & spearman & * & AA \\
\hline B_g_Catenibacterium & C22:4 & 0.643357 & 0.024003 & spearman & * AA \\
\hline B_g_Enhydrobacter & C22:4 & -0.62678 & 0.029178 & spearman & * AA \\
\hline B_g_Smithella & C22:4 & -0.60184 & 0.038407 & spearman & AA
\end{tabular}

\section{Correlation between fatty acid content in the plasma and faecal microbial abundance}

There were positive correlations between the abundances of the Citrobacter_rodentium species and Dysgonomonas_sp._BGC7 species in the bacterial kingdom and the content of stearate. The abundances of the Sphingobacteriia class, Sphingobacteriaceae order, Sphingobacteriales family, and Sphingobacterium_gobiense species were proportional to the contents of 10-heptadecenoate, petroselaidate, nervonoate and trans 11-eicosenoate. The abundances of the first three were also proportional to the contents of linoelaidate, and the latter was related to the contents of eicosenoate, rucate, brassidate and docosadienoate. There was a positive correlation between the abundances of the Catenibacterium genus, Catenibacterium_mitsuokai and Ralstonia_pickettii species and the content of docosatetraenoate. The abundance of Enterobacter_sp._50793107, Lactobacillus_equigenerosi, and Lactobacillus_pobuzihii species and the content of nervonoate showed the same correlation. The abundance of the Enterococcus_phage_EFDG1 species in the viral kingdom was also positively correlated with the contents of transvaccenate, linoelaidate, 7-transnonadecenoate and nervonoate.

In the AA group $ه$ there was a negative correlation between the abundance of microbes in the faeces and fatty acid content in the plasma, which showed a correlation between the abundances of the Desulfosarcina genus, Desulfosarcina_cetonica species, Succinispira genus, Succinispira_mobiliz species and 9 other species in the bacterial kingdom and the content of caproate. There was also a correlation between the content of docosadienoate and the abundances of the Enhydrobacter genus, Enhydrobacter_aerosaccus species, Mycoaviduss genus, Mycoavidus_cysteinexigens species and 5 other species in the bacterial kingdom. Therefore, there was a correlation between the content of isobutyric acid and the abundances of the Citrobacter genus, Citrobacter pasteurii species and Citrobacter sp. MGH106 species. The species abundances of Chloroflexi_bacterium_ GWB2_ 49_20, Kocuria_kristinae, and Lactobacillus_ Equigenerosi were negatively correlated with the content of petroselaidate, the latter two with the content of transvaccenate and the latter with the content of linoelaidate. The species abundances of Chloroflexi_bacterium_GWB2_49_20, Enterobacter_sp._50793107, Lactobacillus_equigenerosi, and Serratia_sp._TEL were negatively correlated with the contents of trans 11-eicosenoate, linoelaidate, brassidate, and erucate, the former 1 with the content of linoelaidate. There were also negative correlations between microbial abundance and the contents of various fatty acids, which showed a correlation between the abundance of the Lactobacillus_equigenerosi species and the contents of palmitelaidate, 10-heptadecenoate, 10-transsheptadecenoate, petroselaidate, transvaccenate, linoelaidate, 7-transnonadecenoate, eicosenoate, trans 11-eicosenoate, erucate, brassidate, docosadienoate, docosatetraenoate, nervonoate, and caproate. There was also a correlation between the abundances of the Cruoricaptor genus and Cruoricaptor_ignavus species and the contents of palmitelaidate, transsheptadecenoate, stearate, and caproate; a correlation between the abundance of the Lactobacillus_pobuzihii species the contents of 7-transnonadecenoate, nervonoate, and isobutyric acid; and a correlation between the abundance of Serratia_sp._TEL and the contents of 11-eicosenoate, trans 11-eicosenoate, erucate, brassidate, docosadienoate, and isobutyric acid. A negative correlation was also found between the species abundance of Enterobacter_sp._50793107 and the contents of trans 11-eicosenoate, erucate, brassidate, nervonoate and isobutyric acid. There was a negative correlation between the genus abundance of Lentzea and the contents of linoelaidate, 7-transnonadecenoate, and 11-eicosenoate; a negative correlation between the species abundance of Chloroflexi_bacterium_GWB2_49_20C and the contents of petroselaidate, linoelaidate, and 11-eicosenoate; a negative correlation between the species abundance of Bifidobacterium_saeculare and the contents of docosatetraenoate and caproate; a negative correlation between the species abundance of Gulbenkiania_indica and the contents of caproate and isobutyric acid; a negative correlation between the species abundance of Kocuria_kristinae and the contents of petroselaidate and transvaccenate; and a negative correlation between the species abundance of Xenorhabdus_innexi and the contents of docosadienoate and isobutyric acid. There was also a negative correlation between microbial abundance in the eukaryotic kingdom and fatty acid content in the plasma, which showed a negative correlation between the genus abundance of Arabidopsis and the content of 11-eicosenoate; and negative correlations between the species abundance of Candida_maltosa and the content of stearate $\mathbb{Z}$ the genus abundance of Cucurbita and the contents of 10-heptadecenoate, petroselaidate, transvaccenate, linoelaidate, docosatetraenoate and the species 
abundance of Cucurbita_moschata and the contents of 10-heptadecenoate, petroselaidate, transvaccenate, linoelaidate, and docosatetraenoate. The abundances of the Nematoda phylum, Enoplea class, Trichinellida order, Trichuridae family, and Trichuris genus were also negatively correlated with the contents of 11-eicosenoate and docosatetraenoate『and the first was correlated with the content of transsheptadecenoate (Tab 13,.Fig 20).

Tab 13. the specific correlation between the microbial abundance and fatty acids in the plasma in AA group(only the top and last 20 showed here) 


\begin{tabular}{|c|c|c|c|c|c|c|}
\hline nameA & nameB & correlation & P.value & method & star & group \\
\hline B_S_Dialister_invisus_CAG:218 & $\mathrm{C} 14: 1 \mathrm{~T}$ & 0.839161 & 0.000643 & spearman & ** & AA \\
\hline B_g_Catenibacterium & $\mathrm{C} 22: 4$ & 0.797203 & 0.0019 & spearman & ** & AA \\
\hline B_s_Catenibacterium_mitsuokai & $\mathrm{C} 22: 4$ & 0.797203 & 0.0019 & spearman & $\star \star$ & AA \\
\hline B_s_Ralstonia_pickettii & $\mathrm{C} 22: 4$ & 0.74081 & 0.005847 & spearman & ** & AA \\
\hline B_s_Sphingobacterium_gobiense & $\mathrm{C} 18: 1 \mathrm{~N} 12 \mathrm{~T}$ & 0.739001 & 0.006033 & spearman & $\star \star$ & AA \\
\hline B_s_Lactobacillus_pobuzihii & $\mathrm{C} 14: 1 \mathrm{~T}$ & 0.734316 & 0.006538 & spearman & $\star \star$ & AA \\
\hline B_s_Citrobacter_rodentium & C18:0 & 0.734266 & 0.006543 & spearman & ** & AA \\
\hline B_s_Sphingobacterium_gobiense & $\mathrm{C} 20: 1$ & 0.727804 & 0.007291 & spearman & ** & AA \\
\hline B_s_Cellulomonas_marina & $\mathrm{C} 14: 1 \mathrm{~T}$ & 0.681607 & 0.014642 & spearman & * & $\mathrm{AA}$ \\
\hline V_s_Enterococcus_phage_EFDG1 & C18:2N6T & 0.679284 & 0.015117 & spearman & * & AA \\
\hline V_s_Enterococcus_phage_EFDG1 & C19:1N12T & 0.668087 & 0.017569 & spearman & * & AA \\
\hline B_s_Dysgonomonas_sp._BGC7 & $\mathrm{C} 18: 0$ & 0.664336 & 0.018453 & spearman & * & AA \\
\hline B_s_Dialister_invisus & $\mathrm{C} 14: 1 \mathrm{~T}$ & 0.65035 & 0.022034 & spearman & * & AA \\
\hline B_s_Sphingobacterium_gobiense & C17:1T & 0.645693 & 0.023332 & spearman & * & AA \\
\hline B_f__Sphingobacteriaceae & $\mathrm{C} 17: 1$ & 0.636364 & 0.026097 & spearman & * & AA \\
\hline B_o_Sphingobacteriales & $\mathrm{C} 17: 1$ & 0.636364 & 0.026097 & spearman & * & AA \\
\hline V_s_Enterococcus_phage_EFDG1 & C18:1N7T & 0.630764 & 0.027866 & spearman & * & AA \\
\hline B_c_Sphingobacteriia & $\mathrm{C} 17: 1$ & 0.629371 & 0.02832 & spearman & * & AA \\
\hline B_f__Sphingobacteriaceae & $\mathrm{C} 18: 1 \mathrm{~N} 12 \mathrm{~T}$ & 0.629371 & 0.02832 & spearman & * & AA \\
\hline B_f__Sphingobacteriaceae & $\mathrm{C} 20: 1 \mathrm{~T}$ & 0.629371 & 0.02832 & spearman & * & AA \\
\hline E_f__Trichuridae & $\mathrm{C} 22: 4$ & -0.76224 & 0.00395 & spearman & ** & $\mathrm{AA}$ \\
\hline E_g_trichuris & $\mathrm{C} 22: 4$ & -0.76224 & 0.00395 & spearman & ** & AA \\
\hline E_o_Trichinellida & $\mathrm{C} 22: 4$ & -0.76224 & 0.00395 & spearman & ** & AA \\
\hline E_g_Cucurbita & $\mathrm{C} 22: 4$ & -0.7642 & 0.003803 & spearman & ** & AA \\
\hline E_s_Cucurbita_moschata & $\mathrm{C} 22: 4$ & -0.7642 & 0.003803 & spearman & $\star *$ & $\mathrm{AA}$ \\
\hline B_s_Serratia_sp._TEL & Isobutyric.acid & -0.77335 & 0.003173 & spearman & ** & AA \\
\hline B_s_Paenibacillus_sp._Aloe- 11 & $\mathrm{C} 6: 0$ & -0.79499 & 0.010445 & spearman & * & $\mathrm{AA}$ \\
\hline B_s_Lactobacillus_equigenerosi & $\mathrm{C} 17: 1 \mathrm{~T}$ & -0.79728 & 0.001897 & spearman & ** & AA \\
\hline B_s_Enterococcus_durans & $\mathrm{C} 6: 0$ & -0.8 & 0.009628 & spearman & ** & AA \\
\hline B_s_Enterobacter_ludwigii & Isobutyric.acid & -0.80911 & 0.001435 & spearman & ** & $\mathrm{AA}$ \\
\hline B_s_Lactobacillus_equigenerosi & C18:2N6T & -0.83643 & 0.000696 & spearman & $\star *$ & $\mathrm{AA}$ \\
\hline B_s_Lactobacillus_equigenerosi & $\mathrm{C} 16: 1 \mathrm{~T}$ & -0.83999 & 0.000627 & spearman & ** & AA \\
\hline B_s_Lactobacillus_equigenerosi & $\mathrm{C} 18: 1 \mathrm{~N} 12 \mathrm{~T}$ & -0.83999 & 0.000627 & spearman & ** & $\mathrm{AA}$ \\
\hline B_s_Lactobacillus_equigenerosi & $\mathrm{C} 22: 1 \mathrm{~N} 9$ & -0.83999 & 0.000627 & spearman & ** & AA \\
\hline B_s_Lactobacillus_equigenerosi & $\mathrm{C} 22: 1 \mathrm{~N} 9 \mathrm{~T}$ & -0.83999 & 0.000627 & spearman & ** & AA \\
\hline B_s_Lactobacillus_equigenerosi & $\mathrm{C} 22: 2$ & -0.84355 & 0.000564 & spearman & ** & $\mathrm{AA}$ \\
\hline B_s_Lactobacillus_equigenerosi & $\mathrm{C} 24: 1$ & -0.85423 & 0.000404 & spearman & ** & $\mathrm{AA}$ \\
\hline B_s_Lactobacillus_equigenerosi & $\mathrm{C} 20: 1 \mathrm{~T}$ & -0.85779 & 0.000359 & spearman & ** & AA \\
\hline B_s_Myxococcus_stipitatus & $\mathrm{C} 6: 0$ & -0.87636 & 0.001935 & spearman & ** & AA \\
\hline B_s_Vibrio_quintilis & $\mathrm{C} 6: 0$ & -0.87636 & 0.001935 & spearman & ** & $\mathrm{AA}$ \\
\hline
\end{tabular}


There was a positive correlation between isovaleric acid, which was elevated in the bone marrow supernatant of individuals in the AA group, and the abundance of genes involved in the type I diabetes mellitus pathway. The lysine degradation pathway was positively correlated with the content of isobutyric acid, which was upregulated in both the peripheral plasma and bone marrow supernatant from the AA group but negatively correlated with stearate, which was downregulated in the peripheral plasma. The downregulated levels of docosadioate, 11-eicosenoate, stearate and docosatetraenoate in the peripheral plasma in the AA group were negatively associated with phenylalanine, tyrosine, and tryptophan biosynthesis, N-glycan biosynthesis, and betaine biosynthesis, respectively. The same correlation was also found between the content of docosadienoate and N-glycan biosynthesis (Tab 14, Fig 21).

Tab 14.the correlation between the abundance of KEGG pathway and fatty acids in plasma and bone marrow supernatant in AA group.

\begin{tabular}{lllllll} 
nameA & nameB & correlation & P.value & method & star & group \\
\hline Betalain biosynthesis & $\mathrm{C} 18: 0$ & 0.58042 & 0.047856 & spearman & $*$ & AABM \\
\hline Lysine degradation & $\mathrm{C} 18: 0$ & 0.608392 & 0.035806 & spearman & * AABM \\
\hline N-Glycan biosynthesis & $\mathrm{C} 20: 1$ & 0.643357 & 0.024003 & spearman & * AABM \\
\hline N-Glycan biosynthesis & $\mathrm{C} 22: 2$ & 0.678322 & 0.015317 & spearman & AABM \\
\hline Phenylalanine, tyrosine and tryptophan biosynthesis & $\mathrm{C} 22: 2$ & -0.58042 & 0.047856 & spearman & * AABM \\
\hline Ubiquinone and other terpenoid-quinone biosynthesis & $\mathrm{C} 22: 4$ & 0.622378 & 0.030676 & spearman & $*$ & AABM \\
\hline Type I diabetes mellitus & Isovaleric.acid & 0.61378 & 0.033762 & spearman & $*$ & AAPB \\
\hline Lysine degradation & Isobutyric.acid & -0.57793 & 0.049049 & spearman & * AAPB
\end{tabular}

\section{Correlations between microbial abundance alterations and KEGG signalling pathways in the AA group}

The abundances of the Mycoavidus genus, Mycoavidus_cysteinexigens species, Achromobacter_sp._ATCC35328 species and Xenorhabdus_innexi species in the bacterial kingdom were negatively correlated with type I diabetes mellitus and positively correlated with the abundances of the Geobacillus_thermodenitrificans species and Kocuria_kristinae species in the bacterial kingdom and the abundance of the Methanobacterium_congolense species in the archaea kingdom. Ubiquinone and other terpenoid-quinone biosynthesis was positively correlated with the abundances of the Catenibacterium genus, Catenibacterium_mitsuokai and Ralstonia_pickettii species and negatively correlated with the abundances of the Desulfosarcina genus, Bifidobacterium_saeculare, Desulfosarcina_cetonica and Megasphaera_massiliensis species in the bacterial kingdom. The abundance of Planctomycetes_bacterium_TMED75 in the bacterial kingdom and Sphingobacterium_gobiense species was positively correlated with N-glycan biosynthesis, which was also negatively correlated with the abundances of Enhydrobacter, the Sulfuricurvum genus, Enhydrobacter_aerosaccus and 6 other species in the bacterial kingdom and the Nematoda phylum, Enoplea class, Trichinellida order, Trichurida family, Arabidopsis, and Trichuris genus in the eukaryotic kingdom. A positive correlation was also found between the abundances of Enhydrobacter and the Sulfuricurvum genera, Enhydrobacter aerosaccus, Enterobacter sp. CC120223-11 and 5 other species in the bacterial kingdom and the phenylalanine, tyrosine and tryptophan biosynthesis signalling pathways, as well as the abundances of the Citrobacter genus, Citrobacter_pasteurii, Citrobacter_rodentium, Citrobacter_sp._MGH106 and 3 other species in the bacterial kingdom and the lysine degradation signalling pathway. The correlation between the abundances of the Cucurbita genus and Cucurbita moschata species in eukaryotes and the phenylalanine, tyrosine and tryptophan biosynthesis signalling pathways was negative. A positive correlation was also found in the betalain biosynthesis signalling pathway and the abundances of the Citrobacter genus, Citrobacter_pasteurii, Citrobacter_rodentium, Citrobacter_sp._MGH106 and 4 other species (Tab 15,Fig 22).

Tab 15.the correlation between the microbial abundance and the KEGG pathway abundance. 


\section{nameA}

B_g_Catenibacterium

B_s_Catenibacterium_mitsuokai

B_s_Ralstonia_pickettii

B_g_Desulfosarcina

B_s_Bifidobacterium_saeculare

B_g_Enhydrobacter

B_s_Desulfosarcina_cetonica

B_g_Sulfuricurvum

B_s_Megasphaera_massiliensis

B_s_Citrobacter_sp._MGH106

B_s_Enhydrobacter_aerosaccus

B_s_Enterobacter_sp._CC120223-11

B_s_Lactobacillus_equigenerosi

B_s_Oceanobacillus_oncorhynchi

B_s_Streptomyces_scabiei

B_s_uncultured_bacterium_5G12

B_s_Planctomycetes_bacterium_TMED75

B_g_Enhydrobacter

B_g_Sulfuricurvum

B_s_Clostridium_sp._3-3

B_s_Sphingobacterium_gobiense

B_g_Citrobacter

B_s_Citrobacter_pasteurii

B_s_Citrobacter_rodentium

B_s_Citrobacter_sp._MGH106

B_s_Enterobacter_ludwigii

B_s_Enterobacter_sp._CC120223-11

B_s_Enhydrobacter_aerosaccus

B_s_Megasphaera_massiliensis

B_s_Oceanobacillus_oncorhynchi

B_s_uncultured_Citrobacter_sp.

B_g_Citrobacter

B_s_Pontibacillus_litoralis

B_s_Citrobacter_pasteurii

B_s_Citrobacter_rodentium
nameB

Ubiquinone and other terpenoid-quinone biosynthesis

Ubiquinone and other terpenoid-quinone biosynthesis

Ubiquinone and other terpenoid-quinone biosynthesis

Ubiquinone and other terpenoid-quinone biosynthesis

Ubiquinone and other terpenoid-quinone biosynthesis

Phenylalanine, tyrosine and tryptophan biosynthesis

Ubiquinone and other terpenoid-quinone biosynthesis

Phenylalanine, tyrosine and tryptophan biosynthesis

Ubiquinone and other terpenoid-quinone biosynthesis

Phenylalanine, tyrosine and tryptophan biosynthesis

Phenylalanine, tyrosine and tryptophan biosynthesis

Phenylalanine, tyrosine and tryptophan biosynthesis

Phenylalanine, tyrosine and tryptophan biosynthesis

Phenylalanine, tyrosine and tryptophan biosynthesis

Phenylalanine, tyrosine and tryptophan biosynthesis

Phenylalanine, tyrosine and tryptophan biosynthesis

$\mathrm{N}$-Glycan biosynthesis

$\mathrm{N}$-Glycan biosynthesis

$\mathrm{N}$-Glycan biosynthesis

$\mathrm{N}$-Glycan biosynthesis

$\mathrm{N}$-Glycan biosynthesis

Lysine degradation

Lysine degradation

Lysine degradation

Lysine degradation

Lysine degradation

Lysine degradation

$\mathrm{N}$-Glycan biosynthesis

$\mathrm{N}$-Glycan biosynthesis

$\mathrm{N}$-Glycan biosynthesis

Lysine degradation

Betalain biosynthesis

$\mathrm{N}$-Glycan biosynthesis

Betalain biosynthesis

Betalain biosynthesis

$\begin{array}{lllll}\text { correlation } & \text { P.value } & \text { method } & \text { star } & \text { level } \\ 0.587413 & 0.044609 & \text { spearman } & * & \text { PB } \\ & & & & \\ 0.587413 & 0.044609 & \text { spearman } & * & \text { PB }\end{array}$

0.5926480 .042283 spearman * PB

$\begin{array}{lll}-0.73677 & 0.00627 & \text { spearman * }\end{array}$

$\begin{array}{llll}-0.85315 & 0.000418 & \text { spearman } * \star & \text { PB }\end{array}$

0.6831160 .014339 spearman * PB

$\begin{array}{lll}-0.84355 & 0.000564 \text { spearman ** } \mathrm{PB}\end{array}$

0.6472340 .022896 spearman * $\mathrm{PB}$

$-0.72727 \quad 0.007355$ spearman ** PB

0.6013990 .038588 spearman * PB

0.6831160 .014339 spearman * PB

0.650350 .022034 spearman * $\mathrm{PB}$

0.5979590 .040012 spearman * PB

0.7178630 .008563 spearman ** PB

0.6264340 .029293 spearman * PB

0.6433570 .024003 spearman * PB

0.701820 .010957 spearman * $\mathrm{PB}$

$\begin{array}{lll}-0.73241 & 0.006752 & \text { spearman ** PB }\end{array}$

$\begin{array}{lll}-0.81099 & 0.00137 & \text { spearman } * * \quad P B\end{array}$

-0.706290 .010245 spearman * PB

0.6046380 .037281 spearman * PB

0.7482520 .005124 spearman ** PB

0.7762240 .002993 spearman ** PB

0.5944060 .041521 spearman * PB

0.7692310 .003446 spearman ** $\mathrm{PB}$

0.8811190 .000153 spearman ** PB

0.790210 .002223 spearman ** PB

$\begin{array}{lll}-0.73241 & 0.006752 & \text { spearman ** PB }\end{array}$

$\begin{array}{lll}-0.71329 & 0.009202 & \text { spearman ** PB }\end{array}$

$\begin{array}{lll}-0.78312 & 0.002591 & \text { spearman ** PB }\end{array}$

0.7482520 .005124 spearman ** $\mathrm{PB}$

0.8881120 .000114 spearman ** PB

$\begin{array}{lll}-0.67182 & 0.016722 & \text { spearman * PB }\end{array}$

0.8181820 .001143 spearman ** PB

0.8391610 .000643 spearman ** PB 


\begin{tabular}{|c|c|c|c|c|c|c|}
\hline B_s_Citrobacter_sp._MGH106 & Betalain biosynthesis & 0.867133 & 0.00026 & spearman & ** & PB \\
\hline B_s_uncultured_bacterium_5G12 & N-Glycan biosynthesis & -0.71329 & 0.009202 & spearman & ** & PB \\
\hline B_s_Enterobacter_ludwigii & Betalain biosynthesis & 0.671329 & 0.016831 & spearman & * & PB \\
\hline B_s_Enterobacter_sp._CC120223-11 & Betalain biosynthesis & 0.776224 & 0.002993 & spearman & ** & PB \\
\hline B_s_uncultured_Citrobacter_sp. & Betalain biosynthesis & 0.839161 & 0.000643 & spearman & ** & PB \\
\hline B_s_Yersinia_enterocolitica & Betalain biosynthesis & 0.594406 & 0.041521 & spearman & * & PB \\
\hline E_c_Enoplea & N-Glycan biosynthesis & -0.6014 & 0.038588 & spearman & * & PB \\
\hline E_f__Trichuridae & N-Glycan biosynthesis & -0.6014 & 0.038588 & spearman & * & PB \\
\hline E_g_Arabidopsis & N-Glycan biosynthesis & -0.71288 & 0.009261 & spearman & $\star \star$ & PB \\
\hline E_g_tTrichuris & N-Glycan biosynthesis & -0.6014 & 0.038588 & spearman & * & PB \\
\hline E_o_Trichinellida & $\mathrm{N}$-Glycan biosynthesis & -0.6014 & 0.038588 & spearman & * & PB \\
\hline E_p_Nematoda & N-Glycan biosynthesis & -0.60839 & 0.035806 & spearman & * & PB \\
\hline E_g_Cucurbita & Lysine degradation & -0.57705 & 0.049477 & spearman & * & PB \\
\hline E_s_Cucurbita_moschata & Lysine degradation & -0.57705 & 0.049477 & spearman & * & PB \\
\hline A_s_Methanobacterium_congolense & Type I diabetes mellitus & -0.69406 & 0.012279 & spearman & * & $\mathrm{BM}$ \\
\hline B_g_Mycoavidus & Type I diabetes mellitus & 0.58042 & 0.047856 & spearman & * & $\mathrm{BM}$ \\
\hline B_s_Achromobacter_sp._ATCC35328 & Type I diabetes mellitus & 0.587342 & 0.044641 & spearman & * & $\mathrm{BM}$ \\
\hline B_s_Geobacillus_thermodenitrificans & Type I diabetes mellitus & -0.7866 & 0.002405 & spearman & $\star \star$ & $\mathrm{BM}$ \\
\hline B_s_Kocuria_kristinae & Type I diabetes mellitus & -0.71596 & 0.008825 & spearman & ** & $\mathrm{BM}$ \\
\hline B_s_Mycoavidus_cysteinexigens & Type I diabetes mellitus & 0.58042 & 0.047856 & spearman & * & BM \\
\hline B_s_Xenorhabdus_innexi & Type I diabetes mellitus & 0.585976 & 0.045263 & spearman & * & $\mathrm{BM}$ \\
\hline
\end{tabular}

\section{Discussion}

Aplastic anaemia is defined as pancytopenia with hypocellular bone marrow in the absence of abnormal infiltrate and no increase in reticulin. To diagnose aplastic anaemia, there must be at least two of the following criteria: (i) haemoglobin $<100 \mathrm{~g} / \mathrm{l}$, (ii) platelet count $<50 \times 10^{9} / \mathrm{l}$, and (iii) neutrophil count $<1.5 \times 10^{9} / 1^{13}$. Most cases of aplastic anaemia are characterized as a bone marrow failure disorder caused by immune cells attacking their own haematopoietic stem cells, and the main effector cells identified are CD8+ T cells (also called cytotoxic T cells, CTLs) that express interferon $\mathrm{Y}^{14}$. However, the mechanism of CD8+ T cell activation is not clear. In addition to cytotoxic T cells, AA patients have immune disorders caused by a large number of other immune molecules. Th1 and Th2 cells are upregulated in aplastic anaemia patients, while Tregs (regulatory T cells) are downregulated in both quantity and function, triggering an autoimmune escape mechanism that drives disease progression ${ }^{15,16}$. The decrease in the number of Tregs may be due to infectioninduced Th1 functional inhibition, which increases the inflammatory response and induces the functional impairment of Tregs ${ }^{16}$. Tregs can inhibit CD8+ $T$ cell function by secreting inhibitory cytokines, inducing apoptosis and interacting with antigen-presenting cells ${ }^{17}$. Dendritic cells (DCs), a class of antigenpresenting cells, especially bone marrow-derived mDCs and plasma cell-derived pDCs. play a role in the pathology of aplastic anaemia. AA patients have upregulated numbers of $\mathrm{DCs}$ and high $\mathrm{mDC} / \mathrm{pDC}$ ratios, as well as upregulated levels of $\mathrm{mDC}$ surface costimulatory molecules and pyruvate kinase 2 (PKM2), demonstrating an upregulation of $\mathrm{mDC}$ function ${ }^{18-20}$. Th17 cells are upregulated in the early disease course and positively correlate with the extent of disease activity, having an inverse relationship with the number of Treg cells, and upregulate Th1 cells by secreting IL-17 ${ }^{21}$. In addition, the pathogenesis of aplastic anaemia has been proven to be related to the bone marrow haematopoiesis microenvironment, in which AA patients contain fewer endosteal, vascular, and perivascular cells than healthy controls ${ }^{22}$. Moreover, MSCs from AA patients exhibit weaker proliferative capacity and modulate the bone marrow immune microenvironment by secreting multiple cytokines ${ }^{23}$ while also having a stronger tendency to differentiate into adipocytes ${ }^{24}$, which are increased in the haematopoietic microenvironment and lead to the inhibition of HSC differentiation and development ${ }^{25}$. In conclusion, aplastic anaemia is an immune disease with a complicated pathogenesis; however, the factors involved in the onset of aplastic anaemia are unclear. Our study revealed the role of lipid metabolism in the pathogenesis of aplastic anaemia through a large number of correlation analyses between macrogenes and lipid metabonomics and showed that Citrobacter rodentium may act as a motile factor in aplastic anaemia.

The species abundances of Citrobacter pasteurii, Citrobacter sp. MGH, and Citrobacter rodentium in the Citrobacter genus in the bacterial kingdom were downregulated in the AA group and had a positive correlation and negative correlation with the contents of stearate and isobutyric acid, respectively(Fig 23). While lysine degradation and the betalain biosynthesis pathway were positively correlated with Citrobacter species abundance, the former had a positive correlation and negative correlation with the contents of stearate and isobutyric acid in the plasma, respectively. Furthermore, the isobutyric acid content increased while the stearic acid (C18:0) content was decreased in the AA group. Citrobacter has been identified as a microorganism that can cause intestinal

Page 16/38 
inflammation; thus, Citrobacter rodentium is inextricably linked to human immune cells, especially dendritic cells and CD4+ T cells ${ }^{26}$. Citrobacter koseri, a subspecies of Citrobacter, stimulates dendritic cells to induce IL-33 through massive ATP production ${ }^{27}$. Plasmacytoid dendritic cells (pDCs) are the main dendritic cells upregulated upon stimulation in Citrobacter rodentium infection ${ }^{28}$. Upregulation of the $\mathrm{mDC} / \mathrm{pDC}$ ratio in aplastic anaemia patients has also been validated, whereby the dendritic cell response due to the downregulation of Citrobacter abundance and immune cell changes in the AA group in the barrier coincide ${ }^{29}$. The involvement of short-chain fatty acids can enhance the induction of Th1 and Th17 cells during Citrobacter rodentium infection in mice $^{30}$. In our study, the isobutyric acid content was upregulated in the AA group and negatively correlated with Citrobacter abundance, coinciding with the upregulation of Th17 cells in AA patients. Downregulation of murine Treg cells enhances Citrobacter rodentium susceptibility, whereas infection with Citrobacter rodentium in the intestinal cells of mice lacking Treg cells elicits a strong Th17 response ${ }^{31}$. Moreover, the branched palmitic acid esters of hydroxy stearic acids (PAHSAs), which induce colonic T cell activation and inhibit proinflammatory cytokine and chemokine expression in mice, attenuate dendritic cell activation and subsequent $\mathrm{T}$ cell proliferation and Th1 polarization in vitro ${ }^{32}$. In our study, the stearic acid content and Citrobacter level were downregulated at the same time, and both were positively correlated, in accordance with the conclusion of the above study. Taken together, these results suggest that the unusual presentation of stearic and isobutyric acids in patients with aplastic anaemia is most likely related to its immunopathogenic mechanisms. Although little research has pointed out the correlation between isobutyric acid and human immunity, studies on short-chain fatty acids (SCFAs) derived from microbes are not rare. Studies have shown that SCFAs promote cell metabolism and enhance the memory potential of activated CD8+ $T$ cells ${ }^{33}$. Patients with aplastic anaemia show downregulation of Treg cells and upregulation of Th17 cells ${ }^{21,34}$. Previous studies have suggested that aplastic anaemia patients are susceptible to Citrobacter, but in our study, Citrobacter abundance was downregulated; however, the immune changes induced by Citrobacter infection were consistent, which suggested that Citrobacter infection might be the driving factor of aplastic anaemia. Citrobacter infection may also be involved in energy metabolism in patients with aplastic anaemia and thus in the gut microenvironment of aplastic anaemia. Citrobacter controls the production of $\mathrm{H} 2 \mathrm{O} 2$ by the NADPH oxidase NOX1 to provide growth conditions for other aerobic bacteria early after infection ${ }^{35}$. The intestinal epithelium absorption of acylcarnitine is impaired by Citrobacter, and then the aerobic metabolism of intestinal epithelial cells is affected ${ }^{36}$. Lysine degradation, which has the same fatty acid correlation with Citrobacter, is a process of energy metabolism that refers to the conversion of lysine to acetyl-coA. Another closely related biosynthesis pathway of betaine is an independent biosynthesis process that focuses on plants. Betaine is the end product of choline oxidation in the human body. Methionine is involved in the biosynthesis of the gut microenvironment, and its role in the formation of the gut microenvironment is unknown. In conclusion, it is reasonable to hypothesize that Citrobacter and its related fatty acids mainly influence the immunopathogenesis of aplastic anaemia and greatly influence the formation of the intestinal microenvironment; therefore, Citrobacter is very likely to be a pathogenic factor of aplastic anaemia and has great potential and research value in aplastic anaemia.

We also noted a decrease in the abundance of Nematoda in eukaryotes identified in aplastic anaemia patients and a decrease in the abundances of Enoplea, Trichinellida, Trichuridae, Enterocytozoon and Trichuris within this phylum, which were negatively correlated with the contents of eicosenoate, docosatetraenoate and 10-transsheptadecenoate in the plasma. In addition, the number of Mycoavidus species ${ }^{37}$ in the nematode-related bacterial community was decreased and negatively correlated with docosadienoate and docosatetraenoate. Previous studies have shown that nematodes are primarily associated with unsaturated fatty acids, consistent with our findings. The nematode Caenorhabditis elegans stores unsaturated fatty acids in droplets in its subcutaneous and intestinal cells ${ }^{38}$. Additionally, 18-carbon PUFAs affect basal innate immune function, the p38 MAP kinase pathway and the transcription of fat-3-regulated genes through the nematode Caenorhabditis elegans to modulate intestinal infection and the expression of genes involved in the stress response, thereby affecting the organism's ability to defend against bacterial infection ${ }^{39}$. Helminth-induced chronic infection can increase anti-inflammatory cytokine secretion and suppress Treg cell activity and increase short-chain fatty acid (SCFA) production ${ }^{40}$. The loss of C. elegans fat-1 expression inhibits lipid droplet formation and selectively disrupts peroxisomes and apical endosomes. Lipid analysis in fat-1-deficient nematodes revealed a significant reduction in heptadecaenoic acid, while other major FAs were unaffected ${ }^{41}$. Caenorhabditis elegans intestinal colonization causes protein homeostasis disruption, which can be improved by butyrate ${ }^{42}$. Two peptides, ACAN1 and NAK1, derived from the nematode phylum, have immunomodulatory functions and can inhibit the proliferation of CD4+ T cells and the production of IL-2 and TNF ${ }^{43}$. Therefore, we can conclude that the nematode phylum acts as a mediator in the human body. On the one hand, the nematode phylum can directly participate in the synthesis and storage of fatty acids; on the other hand, it serves as the link between fatty acid metabolism and immune regulation. The abundance of nematode phylum microbes in the faeces of patients with aplastic anaemia was decreased, as were the levels of several fatty acids that showed negative correlations with it, although there was a negative correlation between the two. There should be a complex interaction mechanism among them. First, the decline in nematode microbiome levels may be due to an increased proportion of T cells in the peripheral blood of immunocompromised patients with aplastic anaemia. Inhibition of the nematode phyla decreased the colonization of long-chain fatty acids in the intestinal epithelium and increased the contents of long-chain fatty acids in the peripheral blood, such that there was a negative correlation between them. We hypothesize that the decrease in fatty acid contents in the peripheral blood may be due to other microorganisms or microbial associations. Docosadienoate was negatively correlated with $\mathrm{N}$-glycan biosynthesis, and $\mathrm{N}$-glycan biosynthesis was negatively correlated with nematode-related microorganisms. $\mathrm{N}$-glycan biosynthesis is associated with weight loss ${ }^{44}$; in other words, there may be a negative correlation with the biosynthesis of fatty acids, which can confirm the negative change in the fatty acids described above and nematode microbial abundance.

The decrease in microbial abundance of the Catenibacterium genus, including Catenibacterium mitsuokaiin species, in the bacterial kingdom was also noTab. In the AA group, there was a positive correlation between the docosatetraenoate content in both the plasma and supernatant, and the docosatetraenoate content was also positively correlated with downregulated ubiquinone and other terpenoid-quinone biosynthesis pathways. Previous studies have shown that the microbial abundance of this genus is similarly downregulated in inflammatory bowel disease patients ${ }^{45}$ and in aplastic anaemia patients in our study, suggesting that it may have some relevance for immunity. In a study of fructose fermentation by faecal microorganisms in vitro, the abundance of Catenibacterium increased with increasing SCFA contents, especially with the increase in butyric acid ${ }^{46,47}$. A study of the gut flora in obese patients found an elevated microbial abundance of Catenibacterium and high plasma levels of short-chain fatty acids, corroborating the above

Page 17/38 
findings ${ }^{48}$. As mentioned earlier, the impact of short-chain fatty acid synthesis on the immune response is gradually being recognized. Valeric acid and butyric acid enhance the antitumour activity of cytotoxic T lymphocytes (CTLs) and chimeric antigen receptor (car) T cells through metabolic and epigenetic reprogramming ${ }^{49}$. SCFAs promote apoptosis by promoting aryl hydrocarbon receptor (AhR) and hypoxia-inducible factor 1a (HIF1a) expression to upregulate IL-22 production by CD4+ T cells ${ }^{50}$. Microbiota-derived metabolites can modulate the suppressive function of Bregs ${ }^{51}$. Butyrate produced by the intestinal flora regulates antigen presentation and radiotherapy following DC-induced antitumour immune responses ${ }^{52}$. As previously mentioned, these immune cells have been implicated in the pathogenesis of aplastic anaemia ${ }^{53}$. Furthermore, docosatetraenoate is positively correlated with ubiquinone and other terpenoid quinone biosynthesis pathways, and recent studies have shown that ubiquinone and other terpenoid quinone biosynthesis pathways are often linked to the synthesis of unsaturated fatty acids ${ }^{54}$. We speculate that the immune abnormalities caused by the decline in the abundance of Catenibacterium mitsuokai species at the Catenibacterium genus may be involved in the pathogenesis of aplastic anaemia; furthermore, the biotransformation of docosatetraenoate is influenced by ubiquinone and other terpenoid quinone biosynthesis pathways. Docosatetraenoate, a long-chain unsaturated fatty acid that cannot be synthesized in the body, has various functions and is involved in the regulation of inflammation and bone destruction through the cyclooxygenase and lipoxygenase pathways ${ }^{55}$. Long-chain unsaturated fatty acids are also involved in the regulation of bone marrow-derived macrophage activity by regulating long-chain acyl-coenzyme A synthetases (ACSLs) ${ }^{56}$ and have a protective effect on drug-induced bone destruction 57,58 In conclusion, unsaturated fatty acids may be involved in the regulation of the inflammatory response and may affect the bone marrow microenvironment. Therefore, the downregulation of docosatetraenoate may be involved in both immune response enhancement and the process of bone destruction and marrow steatosis in aplastic anaemia patients. Although no specific studies have been performed, we believe that unsaturated fatty acids have immense potential in the study of the pathogenesis of aplastic anaemia.

The abundance of Enhydrobacter species was increased in the AA group and was positively correlated with docosadienoatein in the plasma and docosatetraenoate in bone marrow supernatants. Moreover, the abundance of Enhydrobacter aerosaccus species in the Enhydrobacter genus had a positive correlation with phenylalanine, tyrosine and tryptophan biosynthesis but a negative correlation with $\mathrm{N}$-glycan biosynthesis. Previous studies have shown that the Enhydrobacter genus is also abundant in gastrointestinal metaplasia and associated with carcinoma of the head of pancreas (CHP) ${ }^{59,60}$. In in vitro synthesis experiments, an aminotransferase derived from the bacterium Enhydrobacter catalysed the synthesis of L-phenylalanine by using 3-GABA as an amino donor ${ }^{61}$, as confirmed by our results in patients with aplastic anaemia. Studies have shown that L-phenylalanine metabolism is associated with fatty acid synthesis ${ }^{62}$. II-10 has been shown to directly inhibit the function of CD8+ T cells by increasing the number of $n$-glycan branches to decrease antigenic sensitivity ${ }^{63}$. Additionally, CD8+ cell function was upregulated in patients with aplastic anaemia, and our study showed that the abundance of species in the $\mathrm{n}$-branching glycan biosynthesis pathway was decreased in the AA group, consistent with previous findings. $\mathrm{N}$-glycan biosynthesis is also involved in the migration of bone marrow-derived mesenchymal stem cells ${ }^{64}$. Downregulation of $\mathrm{N}$-glycan biosynthesis may inhibit the migration of MSCs outside the bone marrow in aplastic anaemia patients, thus prompting the MSC source to differentiate into adipocytes directly inside the bone marrow and complete the marrow lipidation process. Although there is no research to prove this hypothesis, we still believe there is great research potential. As previously mentioned, long-chain unsaturated fatty acids may be involved in regulating the immune response and preventing bone destruction. However, here, we believe that longchain unsaturated fatty acids primarily affect the process of steatosis in the bone marrow through their role in the bone marrow microenvironment in aplastic anaemia patients. Enhydrobacter (also associated with the presence of long-chain fatty acids) was positively and negatively associated with two biosynthetic pathways, both of which were positively associated with docosadienoate in the plasma and docosatetraenoate in the bone marrow, respectively. Therefore, it is reasonable to hypothesize that this microbe is more likely to affect the aplastic anaemia process than the immune response.

\section{Conclusion}

Our study is the first time that apply Metagenomic to the study of aplastic anemia.Based on the CG-MS lipid detection technique, we found that there were differences in lipid metabolism between the AA group and the NC group, whether short chain fatty acids or medium and long chain fatty acids; Differences in fatty acid metabolism are reflected both in bone marrow supernatants and peripheral plasma, with more significant differences in long-chain fatty acids in peripheral plasma than in short chain fatty acids in bone marrow supernatants;Most fatty acids, whether medium and long chain fatty acids or short chain fatty acids showed a decreasing trend in the disease group, demonstrating that the metabolism of the disease group as a whole was attenuated;

Microbial metabolic alterations are associated with alterations in fatty acids, not only in short but also in long-chain fatty acids, demonstrating that microbial metabolism similarly affects long-chain fatty acid synthesis and metabolism; The interrelationship between Citrobacter spp घstearic acid (c18:0), isobutyric acid, and lysine degradation and betaine biosynthesis pathway suggests that Citrobacter positively regulated stearic acid biosynthesis and precisely negatively regulated isobutyric acid production by these two pathways. The interrelationship between catenibacterium_mitsuokai species at Catenibacterium, cis-7,10,13,16-docosatetraenoic acid (c22:4), ubiquinone and other terpenoid quinones pathway suggests that Catenibacterium negatively regulate cis-7,10,13,16-docosatetraenoic acid by biosynthesis of ubiquinone and other terpenoid quinones. The intercorrelation between the abundance of Enhydrobacter genus and Enhydrobacter_aerosaccus species, cis-13,16-docosadienoic acid in peripheral plasma, cis-7,10,13,16-docosatetraenoic acid in bone marrow supernatant, tyrosine and tryptophan biosynthesis pathway suggests that the genus aquabacterium is associated with two long-chain fatty acids through tyrosine and tryptophan biosynthetic pathway modulation in the disease group.From the point of view of immunity, citreobacilli plays an important role in the pathogenesis of aplastic anemia. We speculate that it may act as a driving factor for aplastic anemia.From the perspective of lipid metabolism, docosanpoly unsaturated fatty acids are of significant relevance to the microbiota in the patient group, and we speculate that docosanpoly unsaturated fatty acids may serve as mediators of microbiota regulated medium - and long-chain fatty acid metabolism.

Overall, our study not only sheds light on the possibility that Citrobacter infection may function as an aplastic anaemia agent but also revealed the potential role of stearate in the immunopathogenesis of aplastic anaemia. In addition, our study demonstrates the potential roles of 22 unsaturated fatty acids in aplastic anaemia. Unsaturated fats are not only involved in the metabolism of fibroblasts in the bone marrow, thus affecting the formation of the bone

Page $18 / 38$ 
marrow microenvironment and participation in the process of bone marrow steatosis, but may also be involved in the regulation of immune cells in the peripheral blood to participate in disease progression in aplastic anaemia patients. Our study provides insights into the pathogenesis of aplastic anaemia and, more importantly, sheds light on the aetiology of aplastic anaemia.

\section{Abbreviations}




\begin{tabular}{|c|c|}
\hline AA & Aplastic anemia \\
\hline NC & Normal control \\
\hline PCR & Polymerase chain reaction \\
\hline PCA & Principal Component Analysis \\
\hline PCR & Polymerase chain reaction \\
\hline PLS-DA & Partial Least Squares-Discriminant Analysis \\
\hline OPLS-DA & Orthogonal Projections to Latent Structures Discriminant Analysis \\
\hline GC-MS & gas chromatography-mass spectrometry \\
\hline $\mathrm{C} 6: 0$ & Caproate \\
\hline C8:0 & Caprylate \\
\hline C10:0 & Caprate \\
\hline C11:0 & Unndecanoate \\
\hline C12:0 & Laurate \\
\hline C13:0 & Tridecanoate \\
\hline C14:0 & Myristate \\
\hline $\mathrm{C} 14: 1 \mathrm{~T}$ & Myristelaidate \\
\hline C14:1 & Myristoleate \\
\hline C15:0 & Pentadecanoate \\
\hline C15:1T & 10-Transpentadecenoate \\
\hline C15:1 & 10-Pentadecenoate \\
\hline C16:0 & Palmitate \\
\hline C16:1T & Palmitelaidate \\
\hline C16:1 & Palmitoleate \\
\hline C17:0 & Heptadecanoate \\
\hline $\mathrm{C} 17: 1 \mathrm{~T}$ & 10-Transsheptadecenoate \\
\hline C17:1 & 10-Heptadecenoate \\
\hline C18:0 & Stearate \\
\hline C18:1N12T & Petroselaidate \\
\hline C18:1N9T & Elaidate \\
\hline C18:1N7T & Transvaccenate \\
\hline C18:1N12 & Petroselinate \\
\hline C18:1N9C & Oleate \\
\hline C18:1N7 & Vaccenate \\
\hline C18:2N6T & Linoelaidate \\
\hline C19:1N12T & 7-Transnonadecenoate \\
\hline C19:1N9T & 10-Transnonadecenoate \\
\hline C18:2N6 & Linoleate \\
\hline C20:0 & Arachidate \\
\hline C18:3N6 & Gamma Linolenate \\
\hline C20:1T & Trans 11-Eicosenoate \\
\hline C20:1 & 11-Eicosenoate \\
\hline C18:3N3 & Alpha Linolenate \\
\hline C21:0 & Heneicosanoate \\
\hline
\end{tabular}

Page 20/38 


\begin{tabular}{ll} 
C20:2 & 11-14 Eicosadienoate \\
\hline C22:0 & Behenate \\
\hline C20:3N6 & Homogamma Linolenate \\
\hline C22:1N9T & Brassidate \\
\hline C22:1N9 & Erucate \\
\hline C20:3N3 & 11-14-17 Eicosatrienoate \\
\hline C20:4N6 & Arachidonate \\
\hline C23:0 & Tricosanoate \\
\hline C22:2 & Docosadienoate \\
\hline C20:5N3 & Eicosapentaenoate \\
\hline C24:0 & Lignocerate \\
\hline C24:1 & Nervonoate \\
\hline C22:4 & Docosatetraenoate \\
\hline C22:5N6 & Docosapentaenoate \\
\hline C22:5N3 & Docosapentaenoate \\
\hline C22:6N3 & Docosahexaenoate \\
\hline
\end{tabular}

\section{Declarations}

\section{Ethics approval and consent to participate}

This study is approved by the Tianjin Medical University General Hospital Ethical Committee. Ethical NO. IRB2022-WZ-003

\section{Consent for publication}

Not applicable

\section{Availability of data and materials}

All data generated or analysed during this study are included in this published article [and its supplementary information files].

\section{Competing interests}

All authors declare no competing interests.

\section{Funding}

This work was supported by National Natural Science Foundation of China(Grant Nos. 81870101,81870117 and 81800120).

\section{Authors' contributions}

Xiaoyu Zhao and Chaomeng Wang analyzed and interpreted data;Xiaoyu Zhao, Chenchen Liu and Nan Jian performed statistical analysis and wrote the manuscript; Tian Zhang, Shaoxue Ding, Tong Chen collected datas;Xiaoyu Zhao, Yingying Sun, Bingnan Liu, Dan Lu, Yingying Chen and Liping Yang performed research and completed the experimental procedures; Chunyan Liu and Rong Fu are corresponding authors and designed our research.

\section{Acknowledgements}

Thanks for National Natural Science Foundation of China for its financial support, the technical support provided by sequencing company and Experimental site provided by Department of Hematology Laboratory of Tianjin Medical University General Hospital.

\section{References}

1. Young NS. Aplastic Anemia. N Engl J Med. Oct 25 2018;379(17):1643-1656. doi:10.1056/NEJMra1413485

2. Stechschulte LA, Ge C, Hinds TD, Jr., Sanchez ER, Franceschi RT, Lecka-Czernik B. Protein Phosphatase PP5 Controls Bone Mass and the Negative Effects of Rosiglitazone on Bone through Reciprocal Regulation of PPARgamma (Peroxisome Proliferator-activated Receptor gamma) and RUNX2 (Runt-related Transcription Factor 2). J Biol Chem. Nov 18 2016;291(47):24475-24486. doi:10.1074/jbc.M116.752493 
3. Wang FS, Ko JY, Yeh DW, Ke HC, Wu HL. Modulation of Dickkopf-1 attenuates glucocorticoid induction of osteoblast apoptosis, adipocytic differentiation, and bone mass loss. Endocrinology. Apr 2008;149(4):1793-801. doi:10.1210/en.2007-0910

4. F.-Z. Chang QW, Q. Zhang, L.-L. Chang, W. Li. Omega-3 polyunsaturated fatty acid inhibits the malignant progression of hepatocarcinoma by inhibiting the Wnt/ß-catenin pathway. review. European Review for Medical and Pharmacological Sciences. 2018;2018; 22 (14): 4500-4508:4500-4508. doi:10.26355/eurrev_201807_15504

5. Rharass T, Lucas S. MECHANISMS IN ENDOCRINOLOGY: Bone marrow adiposity and bone, a bad romance? Eur J Endocrinol. Oct 1 2018;179(4):R165R182. doi:10.1530/EJE-18-0182

6. Al Saedi A, Bermeo S, Plotkin L, Myers DE, Duque G. Mechanisms of palmitate-induced lipotoxicity in osteocytes. Bone. Oct 2019;127:353-359. doi:10.1016/j.bone.2019.06.016

7. Shao Y, Qi W, Zhang X, et al. Plasma Metabolomic and Intestinal Microbial Analyses of Patients With Severe Aplastic Anemia. Front Cell Dev Biol. 2021;9:669887. doi:10.3389/fcell.2021.669887

8. Derrien M, Vaughan EE, Plugge CM, de Vos WM. Akkermansia muciniphila gen. nov., sp. nov., a human intestinal mucin-degrading bacterium. Int $J$ Syst Evol Microbiol. Sep 2004;54(Pt 5):1469-1476. doi:10.1099/ijs.0.02873-0

9. Frost G, Sleeth ML, Sahuri-Arisoylu M, et al. The short-chain fatty acid acetate reduces appetite via a central homeostatic mechanism. Nat Commun. Apr 29 2014;5:3611. doi:10.1038/ncomms4611

10. de Smit SM, de Leeuw KD, Buisman CJN, Strik D. Continuous n-valerate formation from propionate and methanol in an anaerobic chain elongation open-culture bioreactor. Biotechnol Biofuels. 2019;12:132. doi:10.1186/s13068-019-1468-x

11. Donohoe DR, Collins LB, Wali A, Bigler R, Sun W, Bultman SJ. The Warburg effect dictates the mechanism of butyrate-mediated histone acetylation and cell proliferation. Mol Cell. Nov 30 2012;48(4):612-26. doi:10.1016/j.molcel.2012.08.033

12. van der Hee B, Wells JM. Microbial Regulation of Host Physiology by Short-chain Fatty Acids. Trends Microbiol. Aug 2021;29(8):700-712. doi:10.1016/j.tim.2021.02.001

13. Marsh JC, Ball SE, Cavenagh J, et al. Guidelines for the diagnosis and management of aplastic anaemia. Br J Haematol. Oct 2009;147(1):43-70. doi:10.1111/j.1365-2141.2009.07842.x

14. Young NS, Calado RT, Scheinberg P. Current concepts in the pathophysiology and treatment of aplastic anemia. Blood. Oct 15 2006;108(8):2509-19. doi:10.1182/blood-2006-03-010777

15. Kordasti S, Costantini B, Seidl T, et al. Deep phenotyping of Tregs identifies an immune signature for idiopathic aplastic anemia and predicts response to treatment. Blood. Sep 1 2016;128(9):1193-205. doi:10.1182/blood-2016-03-703702

16. Kordasti S, Marsh J, Al-Khan S, et al. Functional characterization of CD4+ T cells in aplastic anemia. Blood. Mar 1 2012;119(9):2033-43. doi:10.1182/blood-2011-08-368308

17. Grover P, Goel PN, Greene MI. Regulatory T Cells: Regulation of Identity and Function. Front Immunol. 2021;12:750542. doi:10.3389/fimmu.2021.750542

18. Sun Y, Wu C, Liu C, et al. Myeloid dendritic cells in severe aplastic anemia patients exhibit stronger phagocytosis. J Clin Lab Anal. Oct 18 2021:e24063. doi:10.1002/jcla.24063

19. Liu B, Shao Y, Liang X, et al. CTLA-4 and HLA-DQ are key molecules in the regulation of mDC-mediated cellular immunity by Tregs in severe aplastic anemia. J Clin Lab Anal. Oct 2020;34(10):e23443. doi:10.1002/jcla.23443

20. Liu C, Zheng M, Wang T, et al. PKM2 Is Required to Activate Myeloid Dendritic Cells from Patients with Severe Aplastic Anemia. Oxid Med Cell Longev. 2018;2018:1364165. doi:10.1155/2018/1364165

21. de Latour RP, Visconte V, Takaku T, et al. Th17 immune responses contribute to the pathophysiology of aplastic anemia. Blood. Nov 18 2010;116(20):4175-84. doi:10.1182/blood-2010-01-266098

22. Wu L, Mo W, Zhang Y, et al. Impairment of hematopoietic stem cell niches in patients with aplastic anemia. Int J Hematol. Dec 2015;102(6):645-53. doi:10.1007/s12185-015-1881-2

23. Gao F, Chiu SM, Motan DA, et al. Mesenchymal stem cells and immunomodulation: current status and future prospects. Cell Death Dis. Jan 21 2016;7:e2062. doi:10.1038/cddis.2015.327

24. Chao YH, Peng CT, Harn HJ, Chan CK, Wu KH. Poor potential of proliferation and differentiation in bone marrow mesenchymal stem cells derived from children with severe aplastic anemia. Ann Hematol. Jul 2010;89(7):715-23. doi:10.1007/s00277-009-0892-6

25. Chao YH, Tsai C, Peng CT, et al. Cotransplantation of umbilical cord MSCs to enhance engraftment of hematopoietic stem cells in patients with severe aplastic anemia. Bone Marrow Transplant. Oct 2011;46(10):1391-2. doi:10.1038/bmt.2010.305

26. Yang W, Liu H, Xu L, et al. GPR120 Inhibits Colitis Through Regulation of CD4(+) T Cell Interleukin 10 Production. Gastroenterology. Sep 16 2021;doi:10.1053/j.gastro.2021.09.018

27. Kataoka H, Mori T, Into T. Citrobacter koseri stimulates dendritic cells to induce IL-33 expression via abundant ATP production. J Med Microbiol. Mar 2021;70(3)doi:10.1099/jmm.0.001303

28. Poysti S, Silojarvi S, Toivonen R, Hanninen A. Plasmacytoid dendritic cells regulate host immune response to Citrobacter rodentium induced colitis in colon-draining lymph nodes. Eur J Immunol. Mar 2021;51(3):620-625. doi:10.1002/eji.202048714

29. Zonghong S, Meifeng T, Huaquan W, et al. Circulating myeloid dendritic cells are increased in individuals with severe aplastic anemia. Int $J$ Hematol. Feb 2011;93(2):156-162. doi:10.1007/s12185-010-0761-z 
30. Park J, Kim M, Kang SG, et al. Short-chain fatty acids induce both effector and regulatory T cells by suppression of histone deacetylases and regulation of the mTOR-S6K pathway. Mucosal Immunol. Jan 2015;8(1):80-93. doi:10.1038/mi.2014.44

31. Wang Z, Friedrich C, Hagemann SC, et al. Regulatory T cells promote a protective Th17-associated immune response to intestinal bacterial infection with C. rodentium. Mucosal Immunol. Nov 2014;7(6):1290-301. doi:10.1038/mi.2014.17

32. Lee J, Moraes-Vieira PM, Castoldi A, et al. Branched Fatty Acid Esters of Hydroxy Fatty Acids (FAHFAs) Protect against Colitis by Regulating Gut Innate and Adaptive Immune Responses. J Biol Chem. Oct 14 2016;291(42):22207-22217. doi:10.1074/jbc.M115.703835

33. Bachem A, Makhlouf C, Binger KJ, et al. Microbiota-Derived Short-Chain Fatty Acids Promote the Memory Potential of Antigen-Activated CD8(+) T Cells. Immunity. Aug 20 2019;51(2):285-297 e5. doi:10.1016/j.immuni.2019.06.002

34. Shi J, Ge M, Lu S, et al. Intrinsic impairment of CD4(+)CD25(+) regulatory T cells in acquired aplastic anemia. Blood. Aug 23 2012;120(8):1624-32. doi:10.1182/blood-2011-11-390708

35. Miller BM, Liou MJ, Zhang LF, et al. Anaerobic Respiration of NOX1-Derived Hydrogen Peroxide Licenses Bacterial Growth at the Colonic Surface. Cell Host Microbe. Dec 9 2020;28(6):789-797 e5. doi:10.1016/j.chom.2020.10.009

36. Smith SA, Ogawa SA, Chau L, et al. Mitochondrial dysfunction in inflammatory bowel disease alters intestinal epithelial metabolism of hepatic acylcarnitines. J Clin Invest. Jan 4 2021;131(1)doi:10.1172/JCl133371

37. Palomares-Rius JE, Gutierrez-Gutierrez C, Mota M, et al. 'Candidatus Xiphinematincola pachtaicus' gen. nov., sp. nov., an endosymbiotic bacterium associated with nematode species of the genus Xiphinema (Nematoda, Longidoridae). Int J Syst Evol Microbiol. Jul 2021;71(7)doi:10.1099/ijsem.0.004888

38. Bouyanfif A, Jayarathne S, Koboziev I, Moustaid-Moussa N. The Nematode Caenorhabditis elegans as a Model Organism to Study Metabolic Effects of omega-3 Polyunsaturated Fatty Acids in Obesity. Adv Nutr. Jan 1 2019;10(1):165-178. doi:10.1093/advances/nmy059

39. Ge H, Li X, Weiszmann J, et al. Activation of G protein-coupled receptor 43 in adipocytes leads to inhibition of lipolysis and suppression of plasma free fatty acids. Endocrinology. Sep 2008;149(9):4519-26. doi:10.1210/en.2008-0059

40. Zaiss MM, Rapin A, Lebon L, et al. The Intestinal Microbiota Contributes to the Ability of Helminths to Modulate Allergic Inflammation. Immunity. Nov 17 2015;43(5):998-1010. doi:10.1016/j.immuni.2015.09.012

41. Li Y, Wang C, Huang Y, et al. C. Elegans Fatty Acid Two-Hydroxylase Regulates Intestinal Homeostasis by Affecting Heptadecenoic Acid Production. Cell Physiol Biochem. 2018;49(3):947-960. doi:10.1159/000493226

42. Walker AC, Bhargava R, Vaziriyan-Sani AS, et al. Colonization of the Caenorhabditis elegans gut with human enteric bacterial pathogens leads to proteostasis disruption that is rescued by butyrate. PLoS Pathog. May 2021;17(5):e1009510. doi:10.1371/journal.ppat.1009510

43. Smallwood TB, Navarro S, Cristofori-Armstrong B, et al. Synthetic hookworm-derived peptides are potent modulators of primary human immune cell function that protect against experimental colitis in vivo. J Biol Chem. Jul 2021;297(1):100834. doi:10.1016/j.jbc.2021.100834

44. Greto VL, Cvetko A, Stambuk T, et al. Extensive weight loss reduces glycan age by altering IgG N-glycosylation. Int J Obes (Lond). Jul 2021;45(7):15211531. doi:10.1038/s41366-021-00816-3

45. Masoodi I, Alshanqeeti AS, Ahmad S, et al. Microbial dysbiosis in inflammatory bowel diseases: results of a metagenomic study in Saudi Arabia. Minerva Gastroenterol Dietol. Sep 2019;65(3):177-186. doi:10.23736/S1121-421X.19.02576-5

46. Liu C, Du P, Cheng Y, et al. Study on fecal fermentation characteristics of aloe polysaccharides in vitro and their predictive modeling. Carbohydr Polym. Mar 15 2021;256:117571. doi:10.1016/j.carbpol.2020.117571

47. Sayago-Ayerdi SG, Zamora-Gasga VM, Venema K. Changes in gut microbiota in predigested Hibiscus sabdariffa $L$ calyces and Agave (Agave tequilana weber) fructans assessed in a dynamic in vitro model (TIM-2) of the human colon. Food Res Int. Jun 2020;132:109036. doi:10.1016/j.foodres.2020.109036

48. Martinez-Cuesta MC, Del Campo R, Garriga-Garcia M, Pelaez C, Requena T. Taxonomic Characterization and Short-Chain Fatty Acids Production of the Obese Microbiota. Front Cell Infect Microbiol. 2021;11:598093. doi:10.3389/fcimb.2021.598093

49. Luu M, Riester Z, Baldrich A, et al. Microbial short-chain fatty acids modulate CD8(+) T cell responses and improve adoptive immunotherapy for cancer. Nat Commun. Jul 1 2021;12(1):4077. doi:10.1038/s41467-021-24331-1

50. Yang W, Yu T, Huang X, et al. Intestinal microbiota-derived short-chain fatty acids regulation of immune cell IL-22 production and gut immunity. Nat Commun. Sep 8 2020;11(1):4457. doi:10.1038/s41467-020-18262-6

51. Rosser EC, Piper CJM, Matei DE, et al. Microbiota-Derived Metabolites Suppress Arthritis by Amplifying Aryl-Hydrocarbon Receptor Activation in Regulatory B Cells. Cell Metab. Apr 7 2020;31(4):837-851 e10. doi:10.1016/j.cmet.2020.03.003

52. Uribe-Herranz M, Rafail S, Beghi S, et al. Gut microbiota modulate dendritic cell antigen presentation and radiotherapy-induced antitumor immune response. J Clin Invest. Jan 2 2020;130(1):466-479. doi:10.1172/JCI124332

53. Javan MR, Saki N, Moghimian-Boroujeni B. Aplastic anemia, cellular and molecular aspects. Cell Biol Int. Aug 18 2021;doi:10.1002/cbin.11689

54. Chen J, Tang H, Li T, et al. Comprehensive Analysis of the Expression, Prognosis, and Biological Significance of OVOLs in Breast Cancer. Int J Gen Med. 2021;14:3951-3960. doi:10.2147/IJGM.S326402

55. Pino AM, Rodriguez JP. Is fatty acid composition of human bone marrow significant to bone health? Bone. Jan 2019;118:53-61. doi:10.1016/j.bone.2017.12.014

56. Kuwata H, Nakatani E, Shimbara-Matsubayashi S, et al. Long-chain acyl-CoA synthetase 4 participates in the formation of highly unsaturated fatty acidcontaining phospholipids in murine macrophages. Biochim Biophys Acta Mol Cell Biol Lipids. Nov 2019;1864(11):1606-1618.

Page 23/38 
doi:10.1016/j.bbalip.2019.07.013

57. Gao B, Han YH, Wang L, et al. Eicosapentaenoic acid attenuates dexamethasome-induced apoptosis by inducing adaptive autophagy via GPR120 in murine bone marrow-derived mesenchymal stem cells. Cell Death Dis. May 26 2016;7:e2235. doi:10.1038/cddis.2016.144

58. Fan CM, Su YW, Howe PR, Xian CJ. Long Chain Omega-3 Polyunsaturated Fatty Acid Supplementation Protects Against Adriamycin and Cyclophosphamide Chemotherapy-Induced Bone Marrow Damage in Female Rats. Int J Mol Sci. Feb 6 2018;19(2)doi:10.3390/ijms19020484

59. Mei QX, Huang CL, Luo SZ, Zhang XM, Zeng Y, Lu YY. Characterization of the duodenal bacterial microbiota in patients with pancreatic head cancer vs. healthy controls. Pancreatology. Jun 2018;18(4):438-445. doi:10.1016/j.pan.2018.03.005

60. Gong J, Li L, Zuo X, Li Y. Change of the duodenal mucosa-associated microbiota is related to intestinal metaplasia. BMC Microbiol. Dec 9 2019;19(1):275. doi:10.1186/s12866-019-1666-5

61. Feng X, Guo J, Zhang R, et al. An Aminotransferase from Enhydrobacter aerosaccus to Obtain Optically Pure beta-Phenylalanine. ACS Omega. Apr 14 2020;5(14):7745-7750. doi:10.1021/acsomega.9b03416

62. Lyu X, Yan K, Chen W, et al. The characterization of metabolites alterations in white adipose tissue of diabetic GK Rats after ileal transposition surgery by an untargeted metabolomics approach. Adipocyte. Dec 2021;10(1):275-284. doi:10.1080/21623945.2021.1926139

63. Smith LK, Boukhaled GM, Condotta SA, et al. Interleukin-10 Directly Inhibits CD8(+) T Cell Function by Enhancing N-Glycan Branching to Decrease Antigen Sensitivity. Immunity. Feb 20 2018;48(2):299-312 e5. doi:10.1016/j.immuni.2018.01.006

64. Alonso-Garcia V, Chaboya C, Li Q, et al. High Mannose N-Glycans Promote Migration of Bone-Marrow-Derived Mesenchymal Stromal Cells. Int J Mol Sci. Sep 29 2020;21(19)doi:10.3390/ijms21197194

\section{Figures}
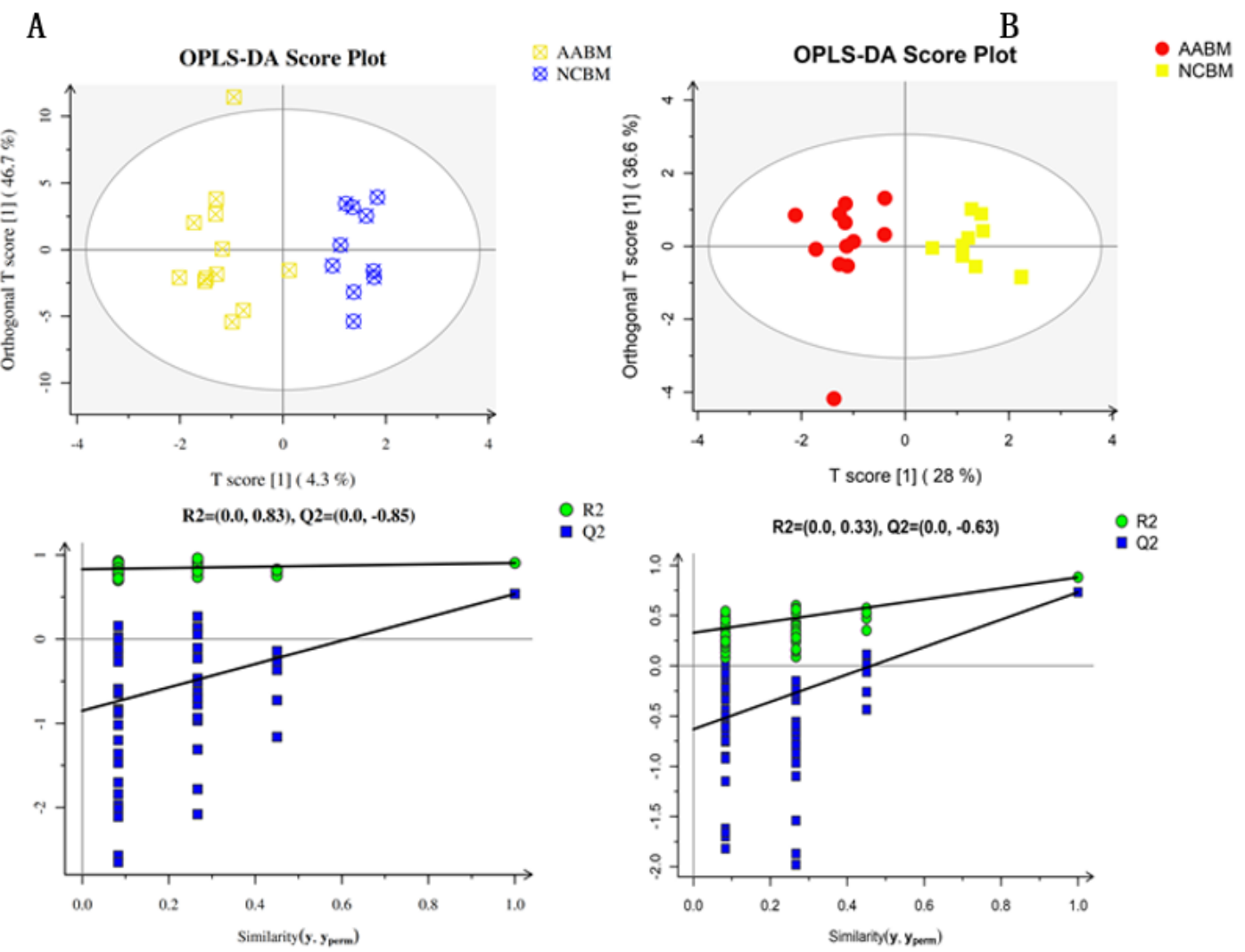

Figure 1

A. OPLS-DA analysis of medium and long-chain fatty acids in the bone marrow;B. OPLS-DA analysis of short-chain fatty acids in the bone marrow 
A

B
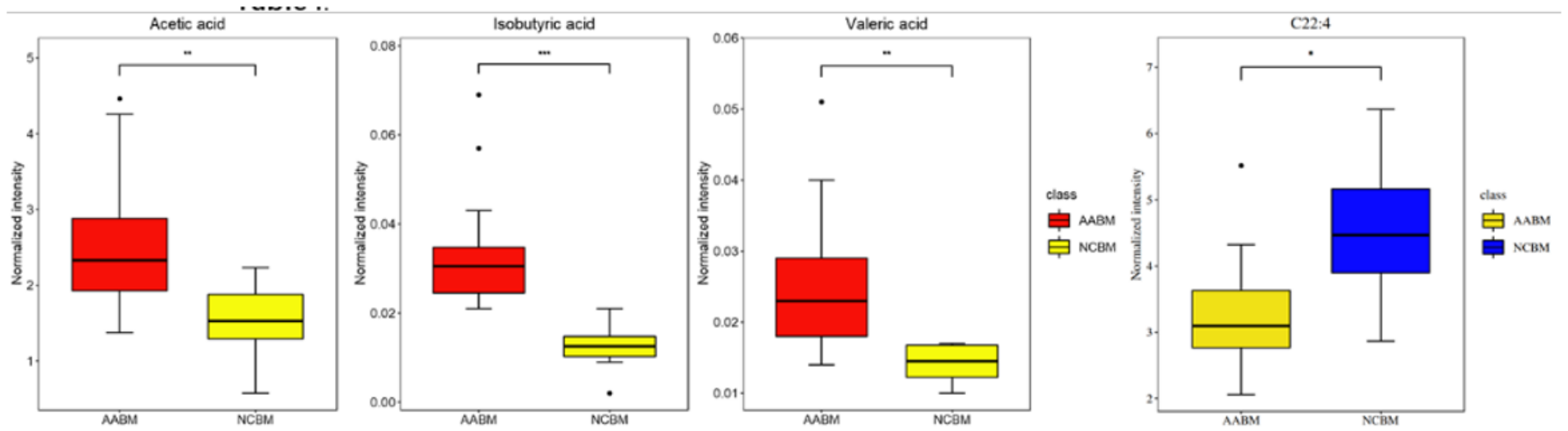

Figure 2

A.3 changed short-chain fatty acids in AA group in the bone marrow .B. docosatetraenoate (C22:4) decreased in AA group in the bone marrow.
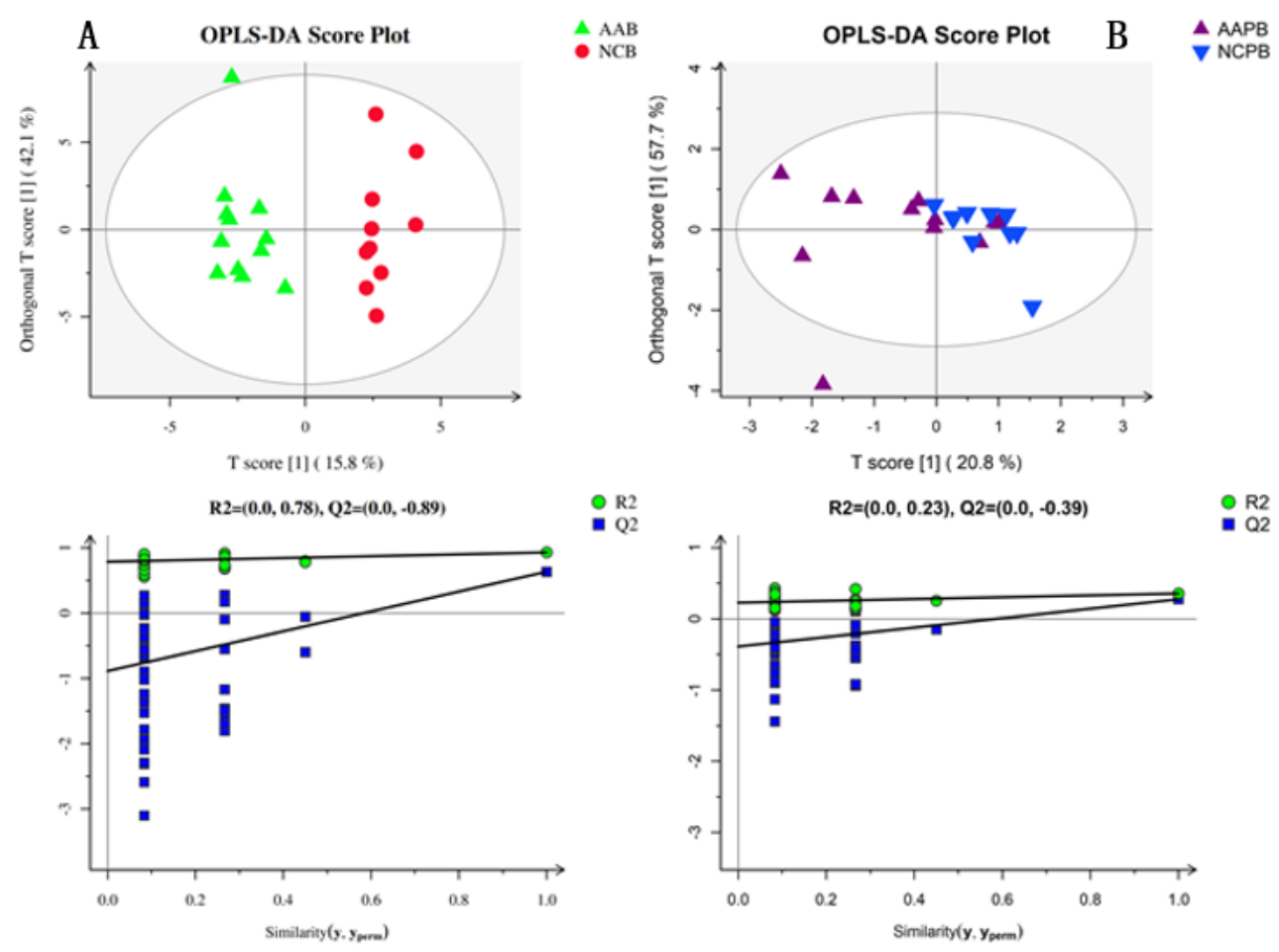

\section{Figure 3}

A. OPLS-DA analysis of medium and long-chain fatty acids in plasma;B. OPLS-DA analysis of short-chain fatty acids in plasma 

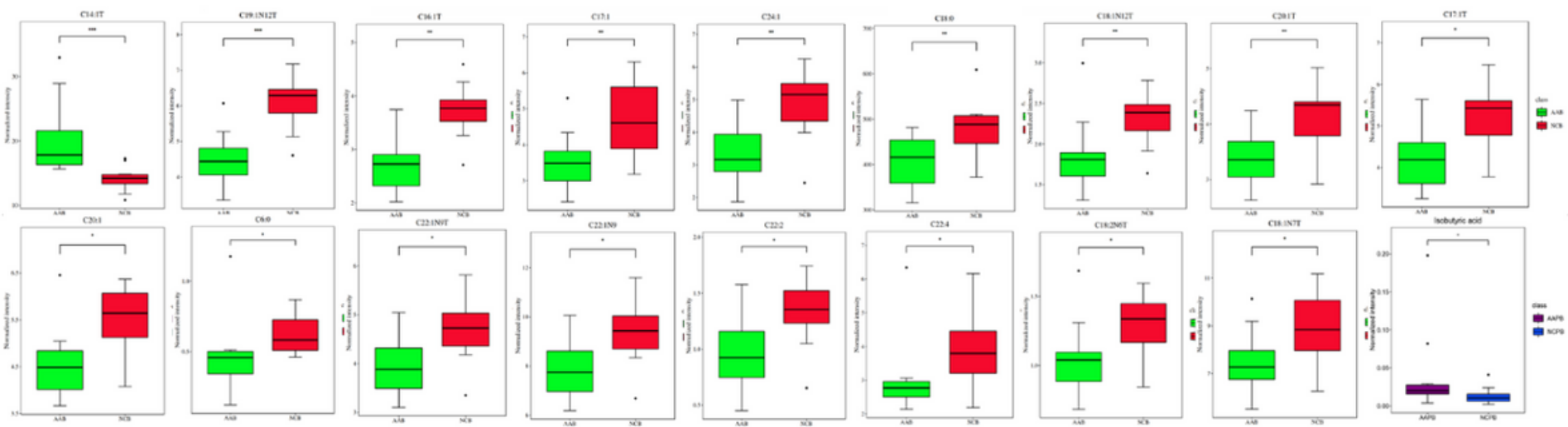

\section{Figure 4}

changed fatty acids(including long-chain fatty acids and short-chain fatty acids) in AA group in plasm.
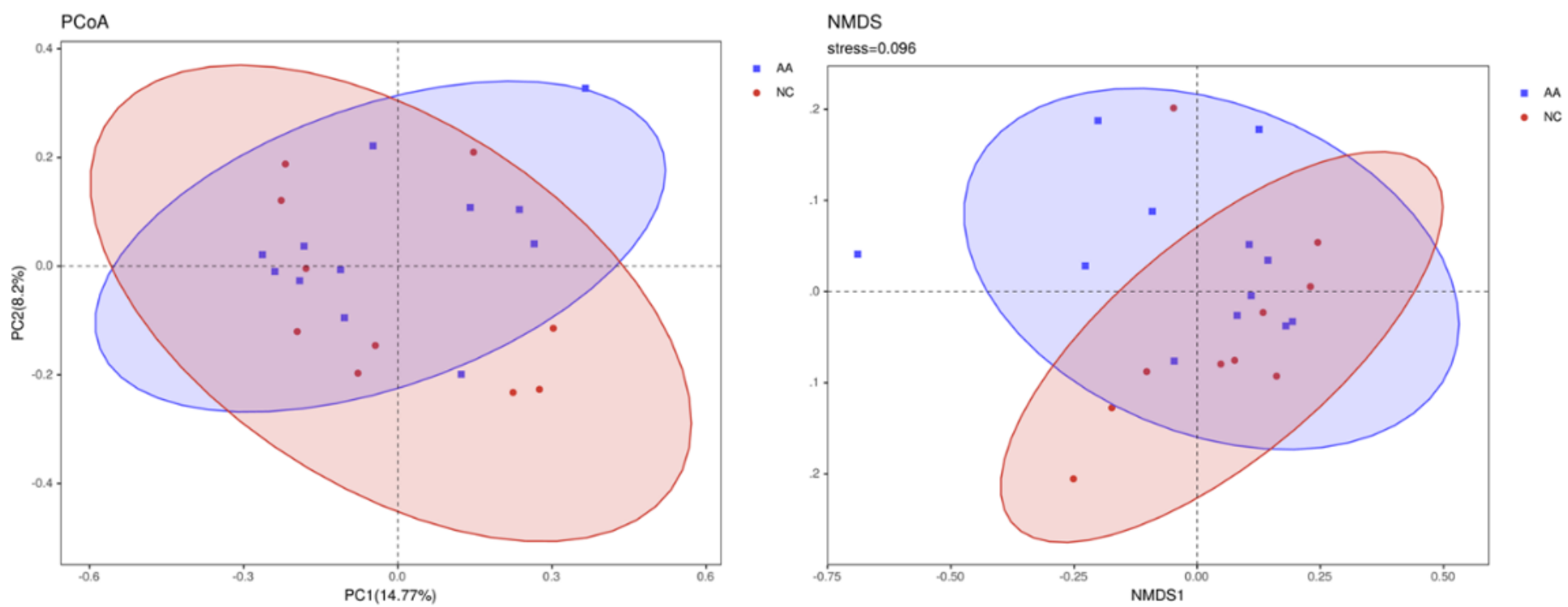

Figure 5

PCoA and NMDS analysis showed no significant community differences between AA and NC groups 

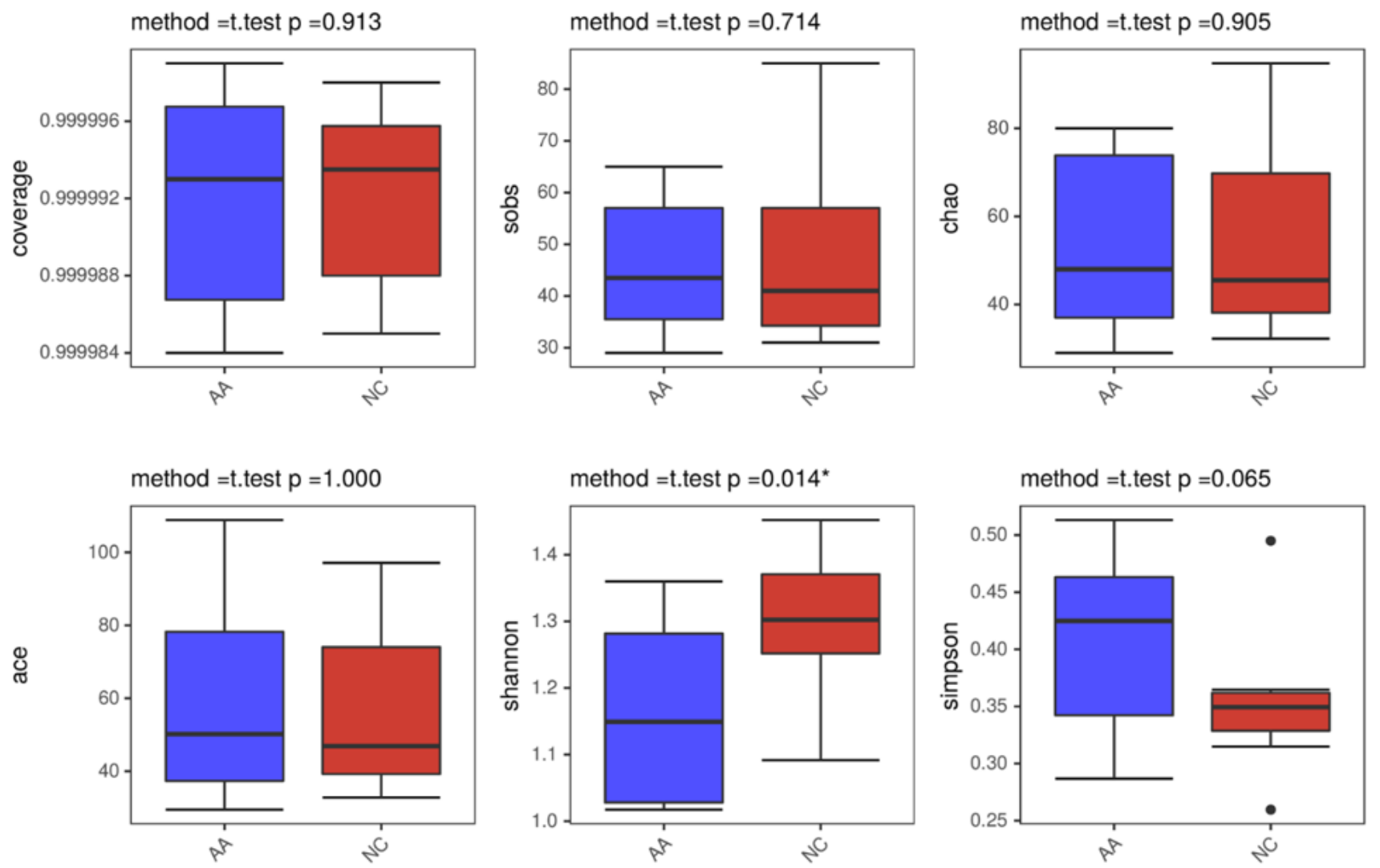

Figure 6

a diversity analysis in phylum level between the NC and AA groups, only the Shannon index shows the significant difference.

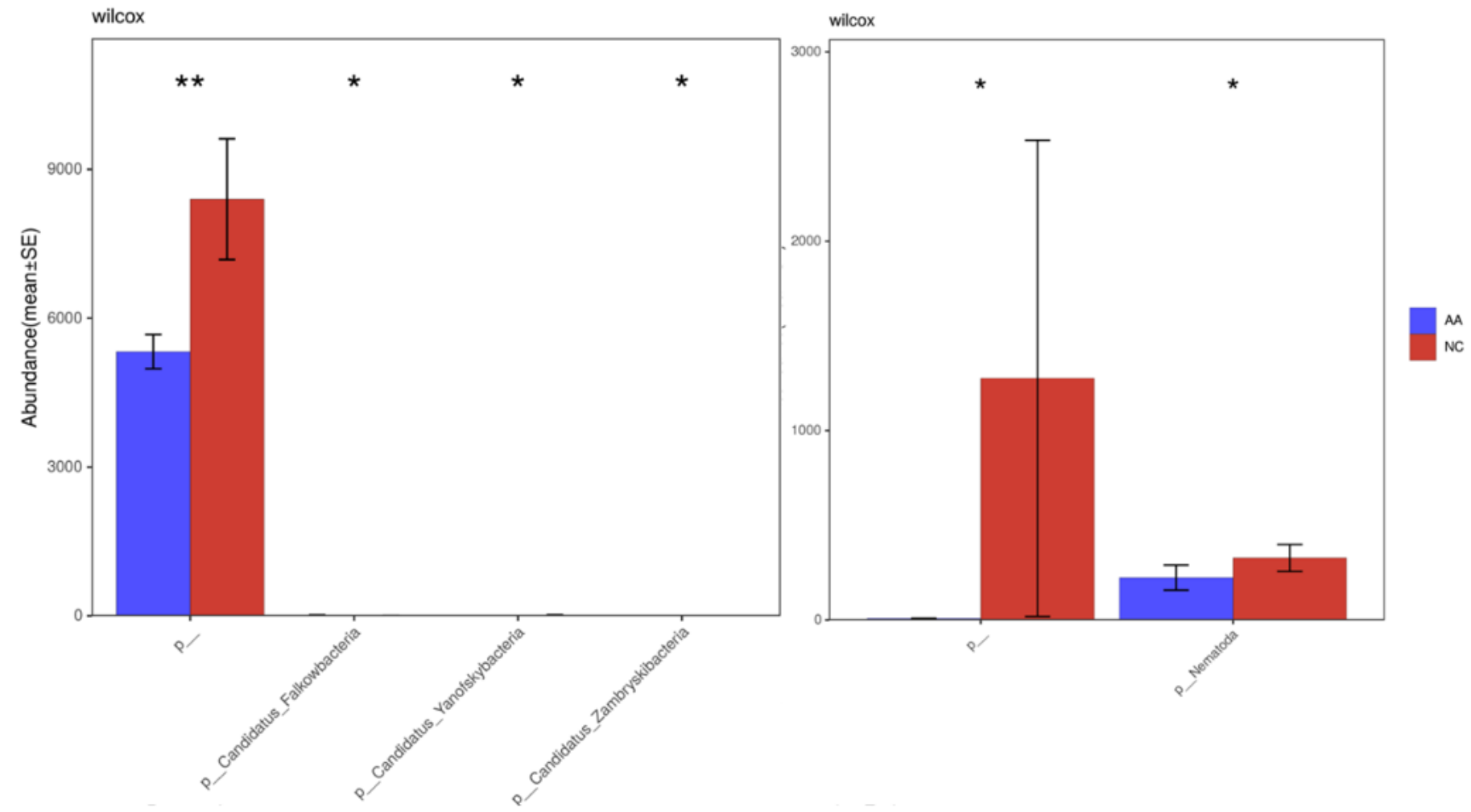

Figure 7 
a.the changed bacterial abundance of AA patients in the phylum level. b. the changed eukaryote abundance of AA patients in the phylum level.
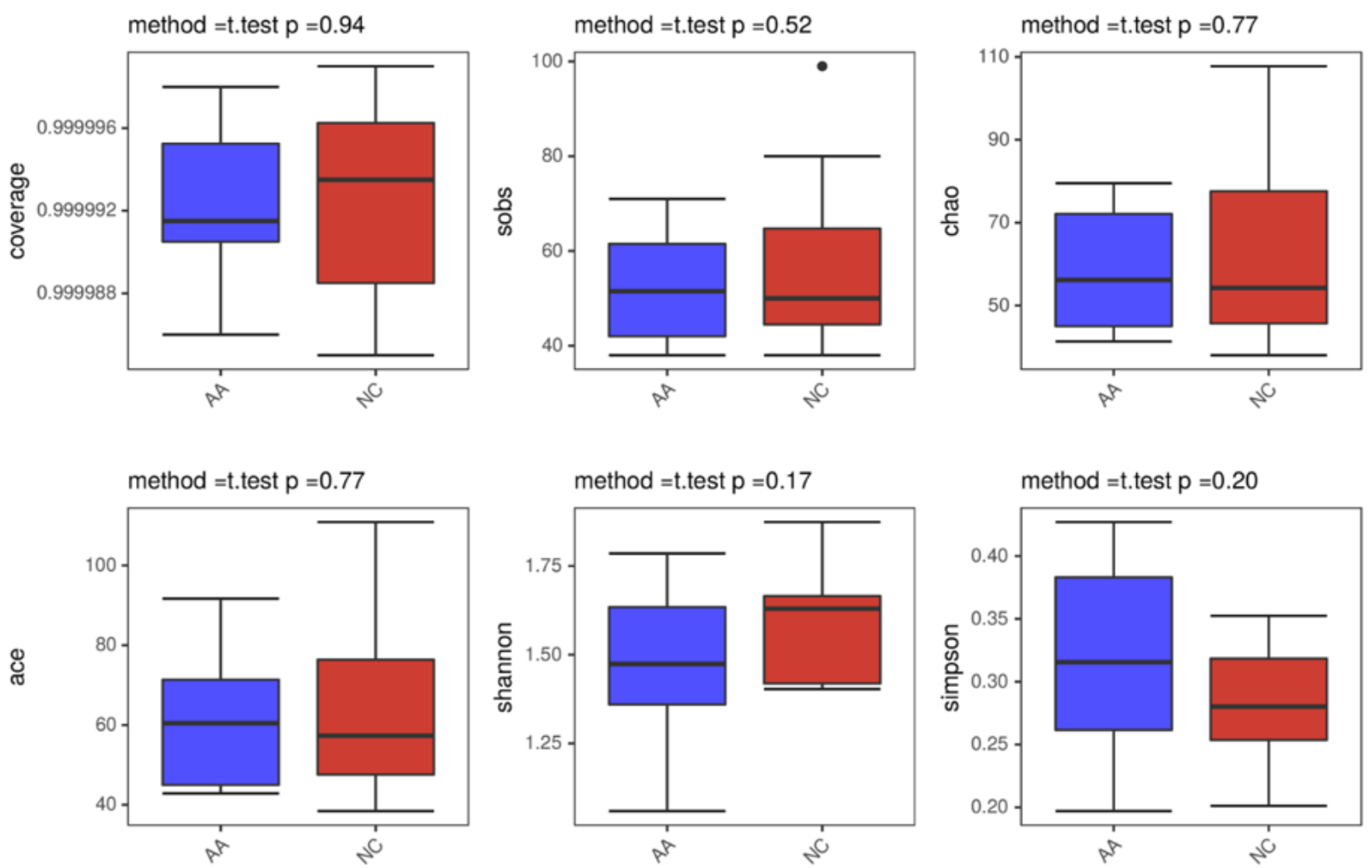

\section{Figure 8}

a diversity analysis in class level between the NC and AA groups, no index shows the significant difference.
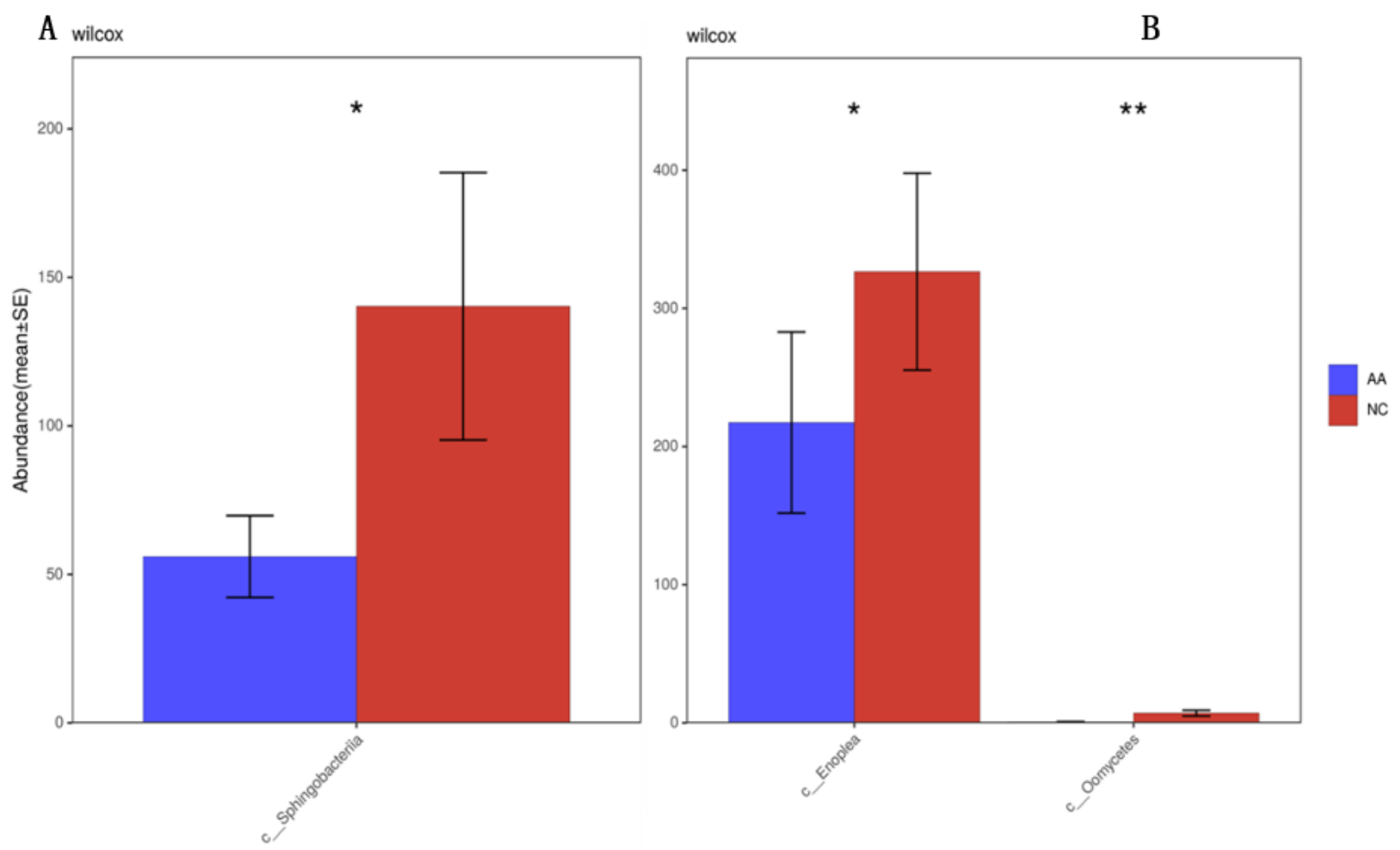

\section{Figure 9}

A. the changed bacterial abundance of AA patients in the class level. B. the changed eukaryote abundance of AA patients in the class level. 

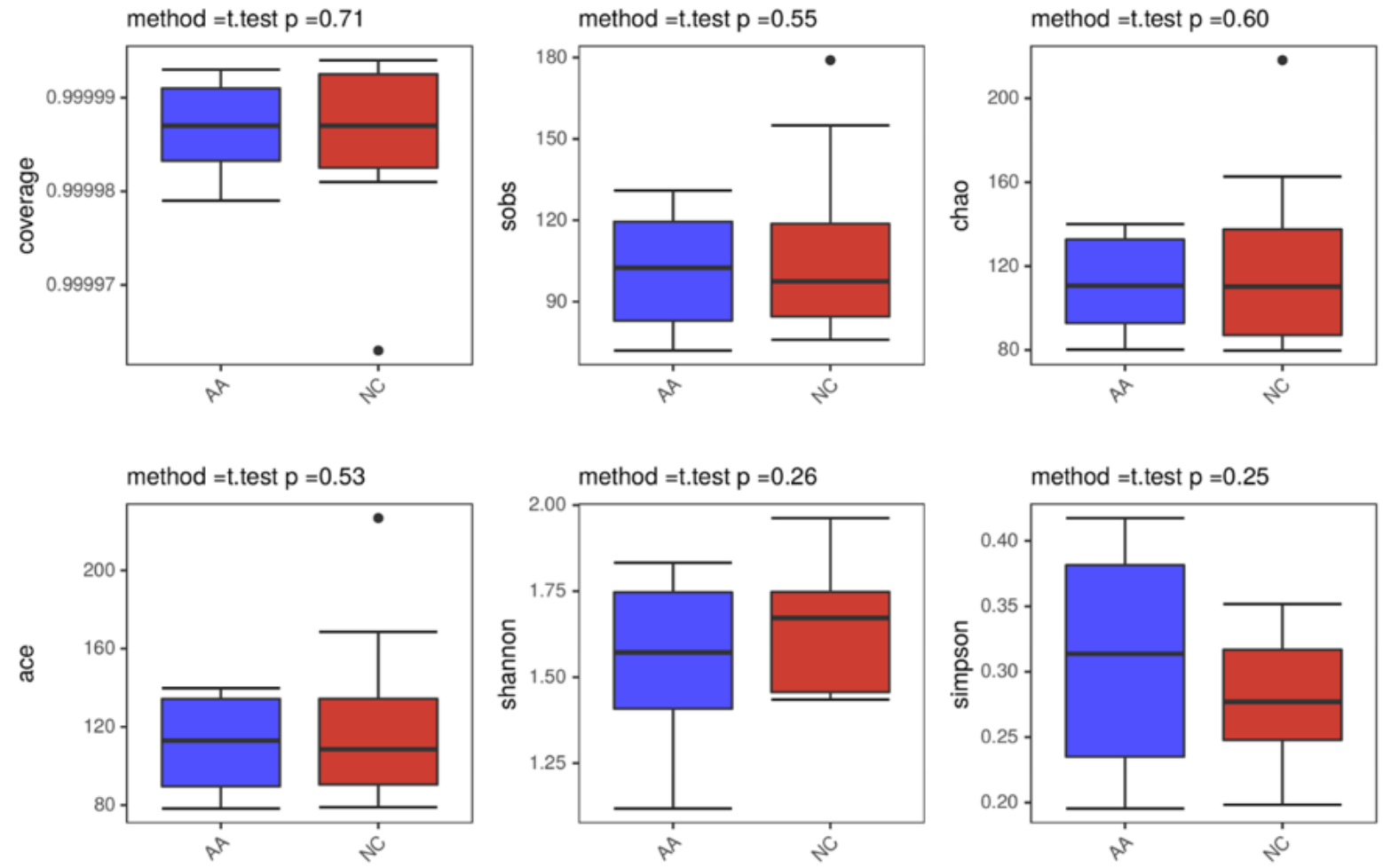

\section{Figure 10}

a diversity analysis in order level between the NC and AA groups, no index shows the significant difference.

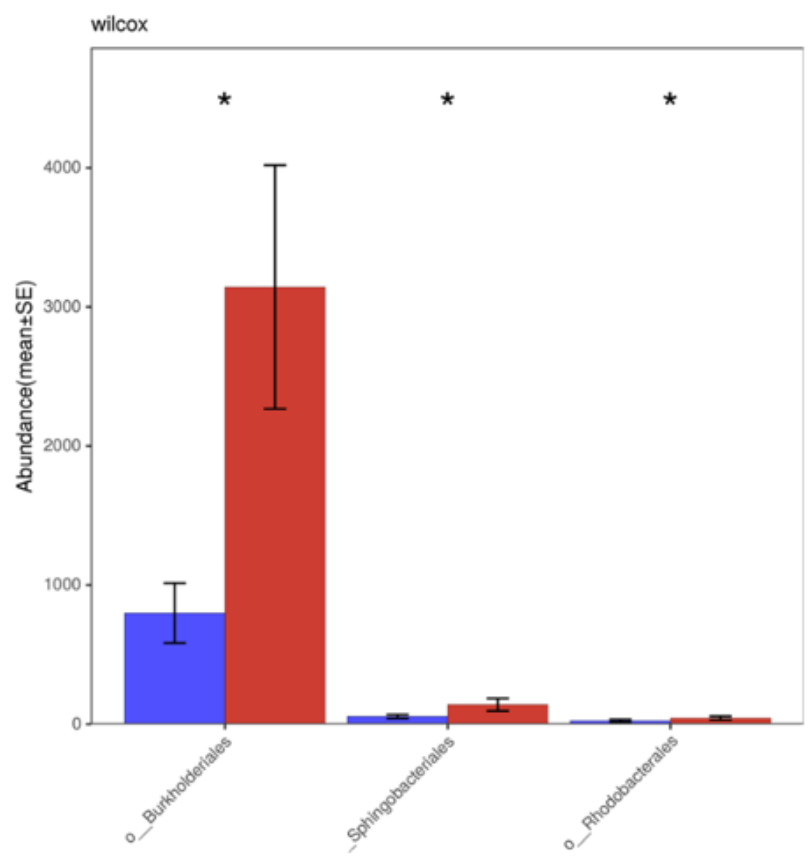

a. Bacteria

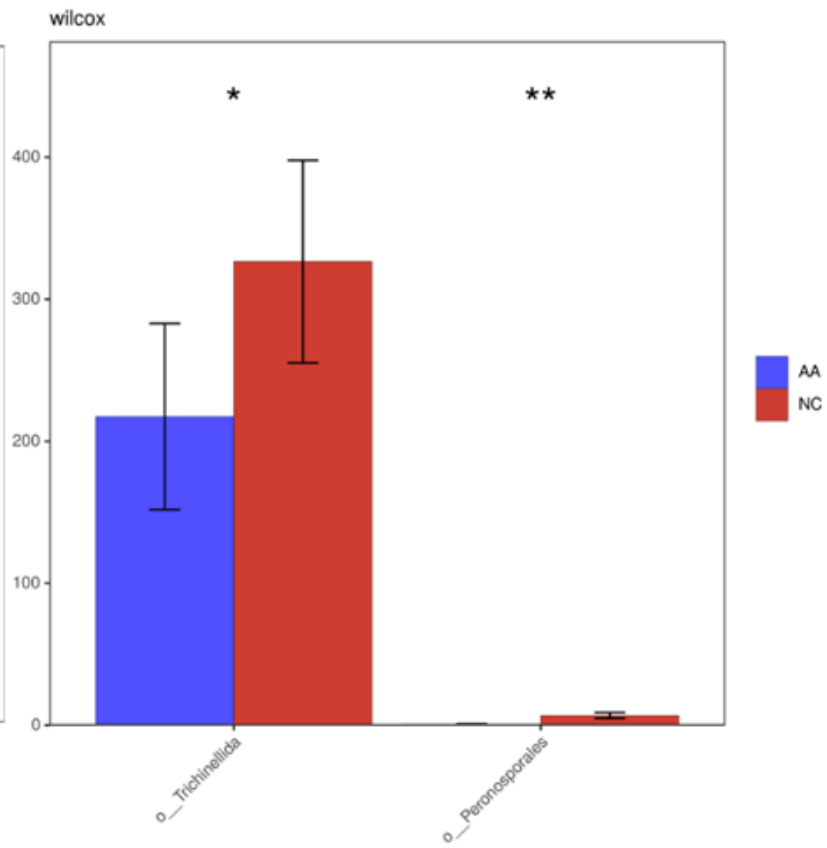

b. Eukaryota

\section{Figure 11}

a. the changed bacterial abundance of AA patients in the order level. b. the changed eukaryote abundance of AA patients in the order level. 

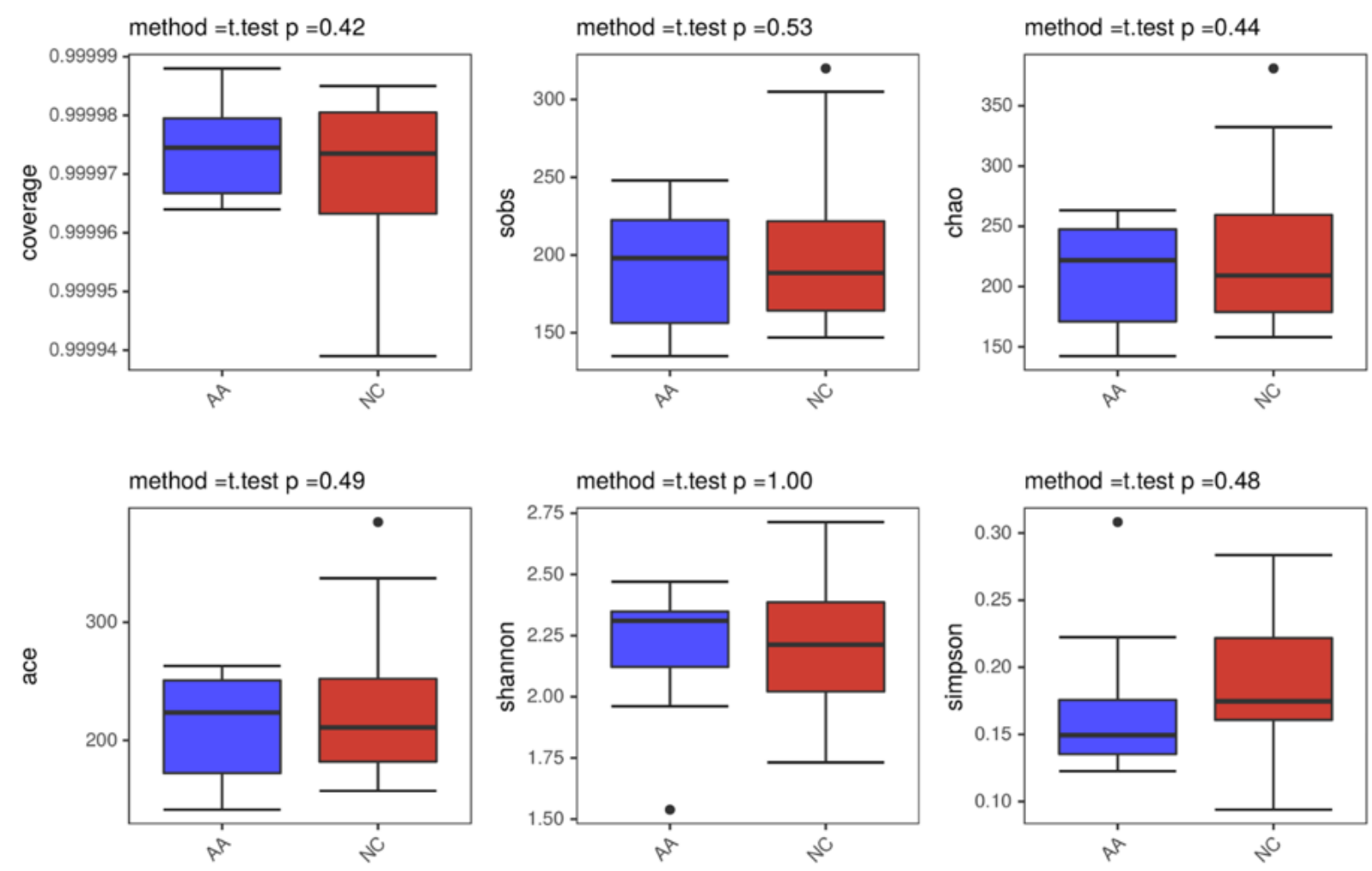

Figure 12

a diversity analysis in family level between the NC and AA groups, no index shows the significant difference

A

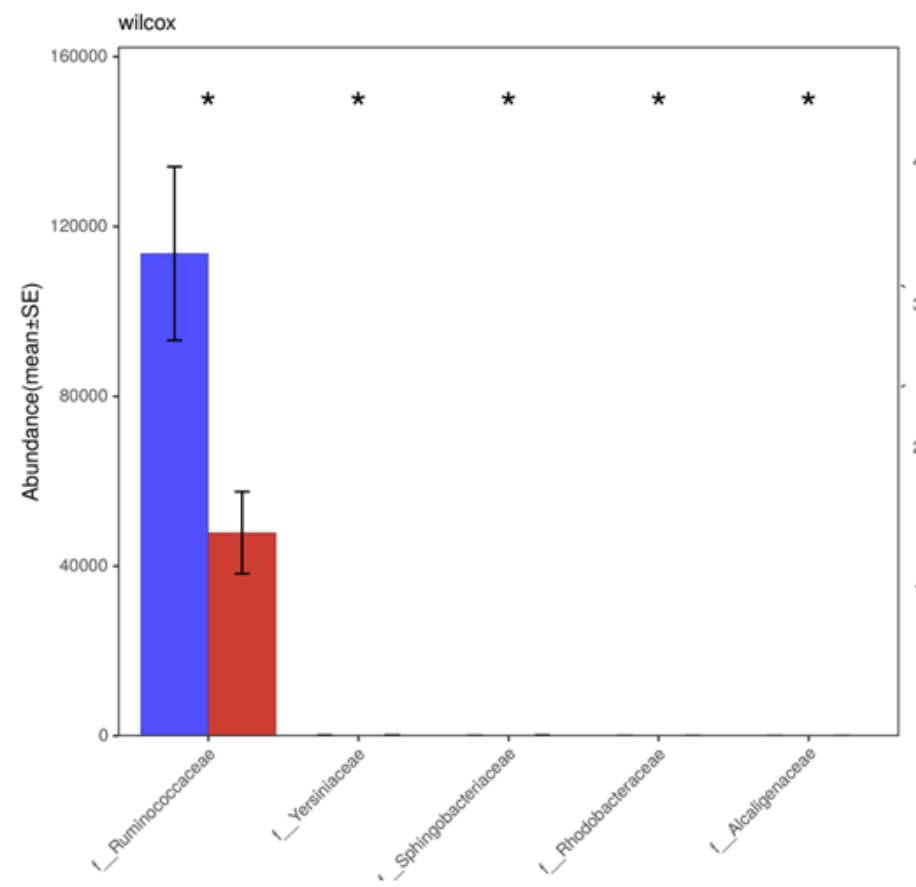

B

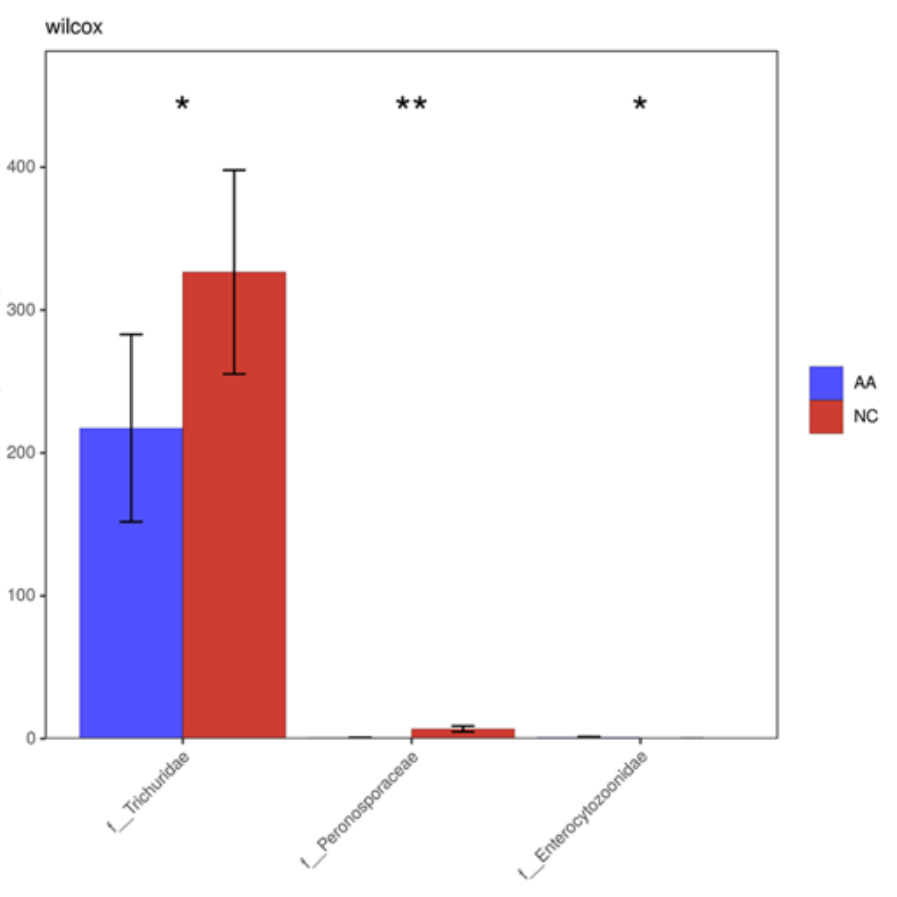

Figure 13

A. the changed bacterial abundance of AA patients in the family level. B. the changed eukaryote abundance of AA patients in the family level. 

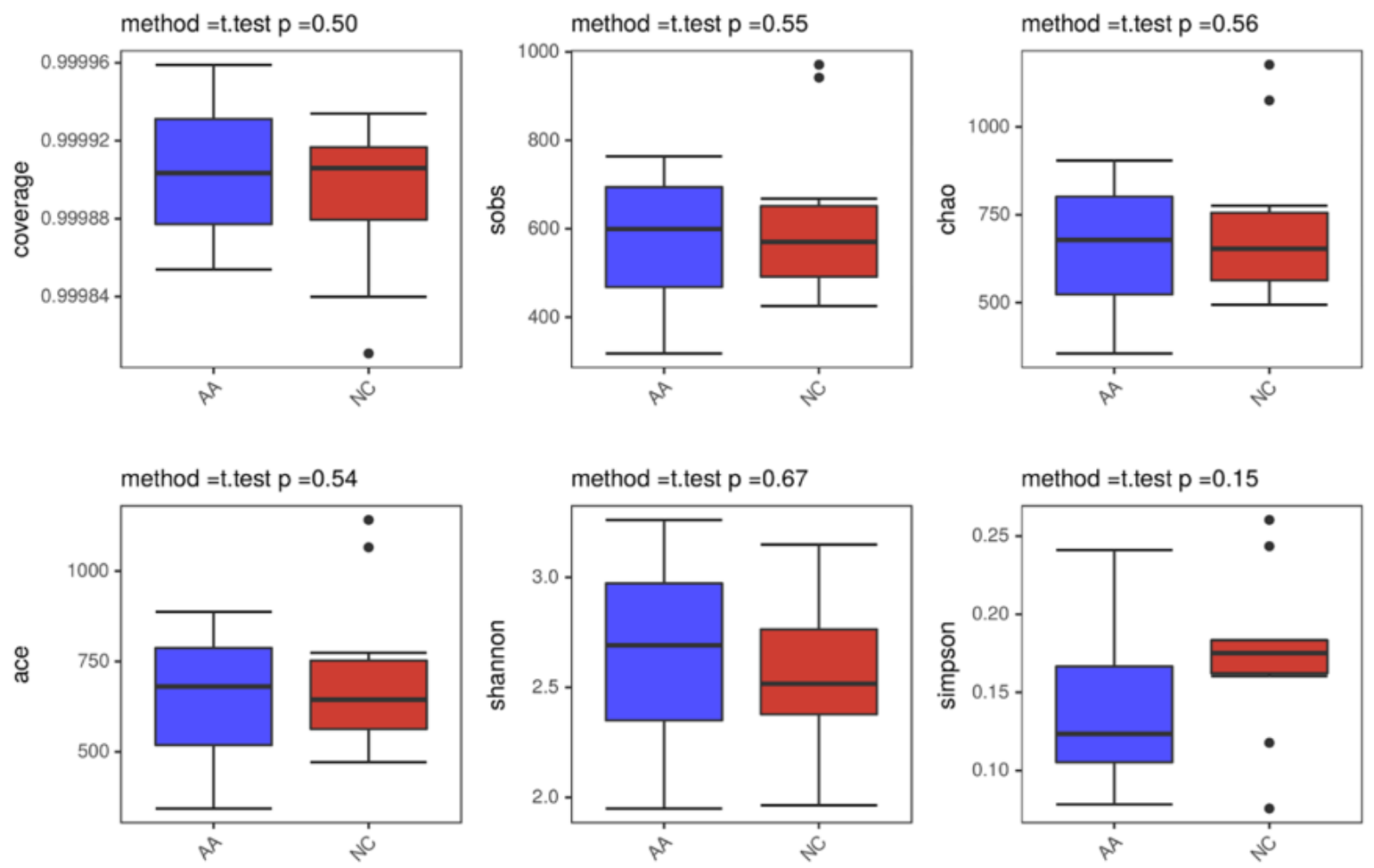

Figure 14

a diversity analysis in family level between the NC and AA groups, no index shows the significant difference

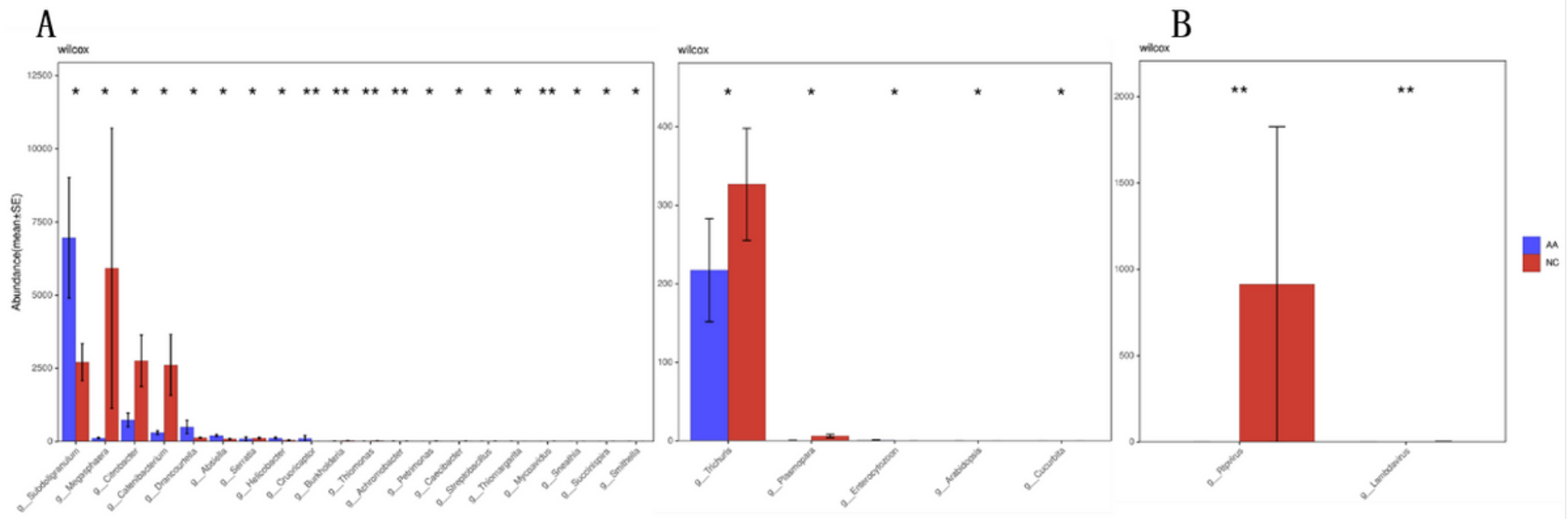

Figure 15

A. the changed bacterial abundance of AA patients in the genus level. B. the changed eukaryote abundance of AA patients in the genus level. C. the changed virus abundance of AA patients in the genus level. 

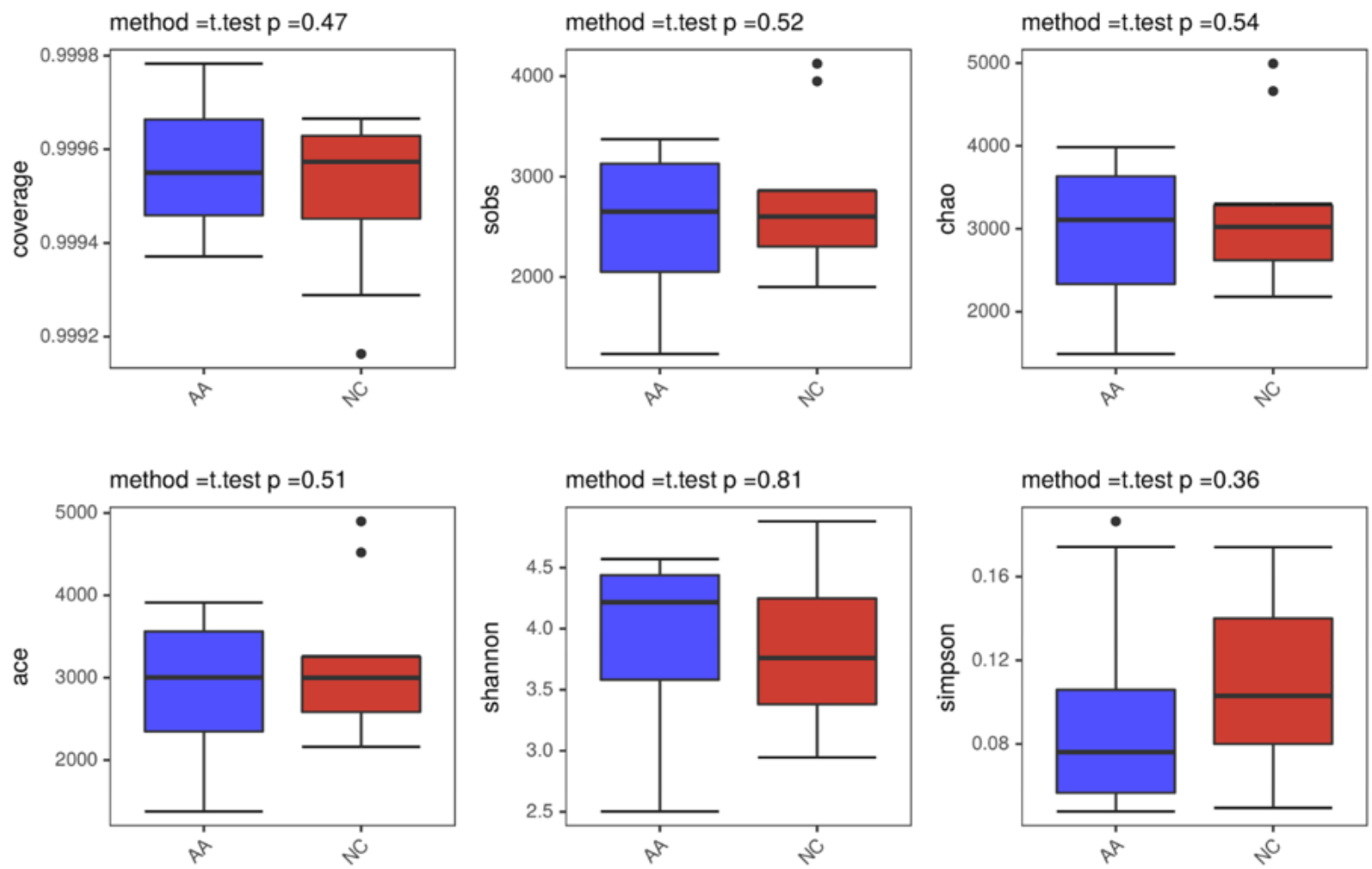

Figure 16

a diversity analysis in family level between the NC and AA groups, no index shows the significant difference 

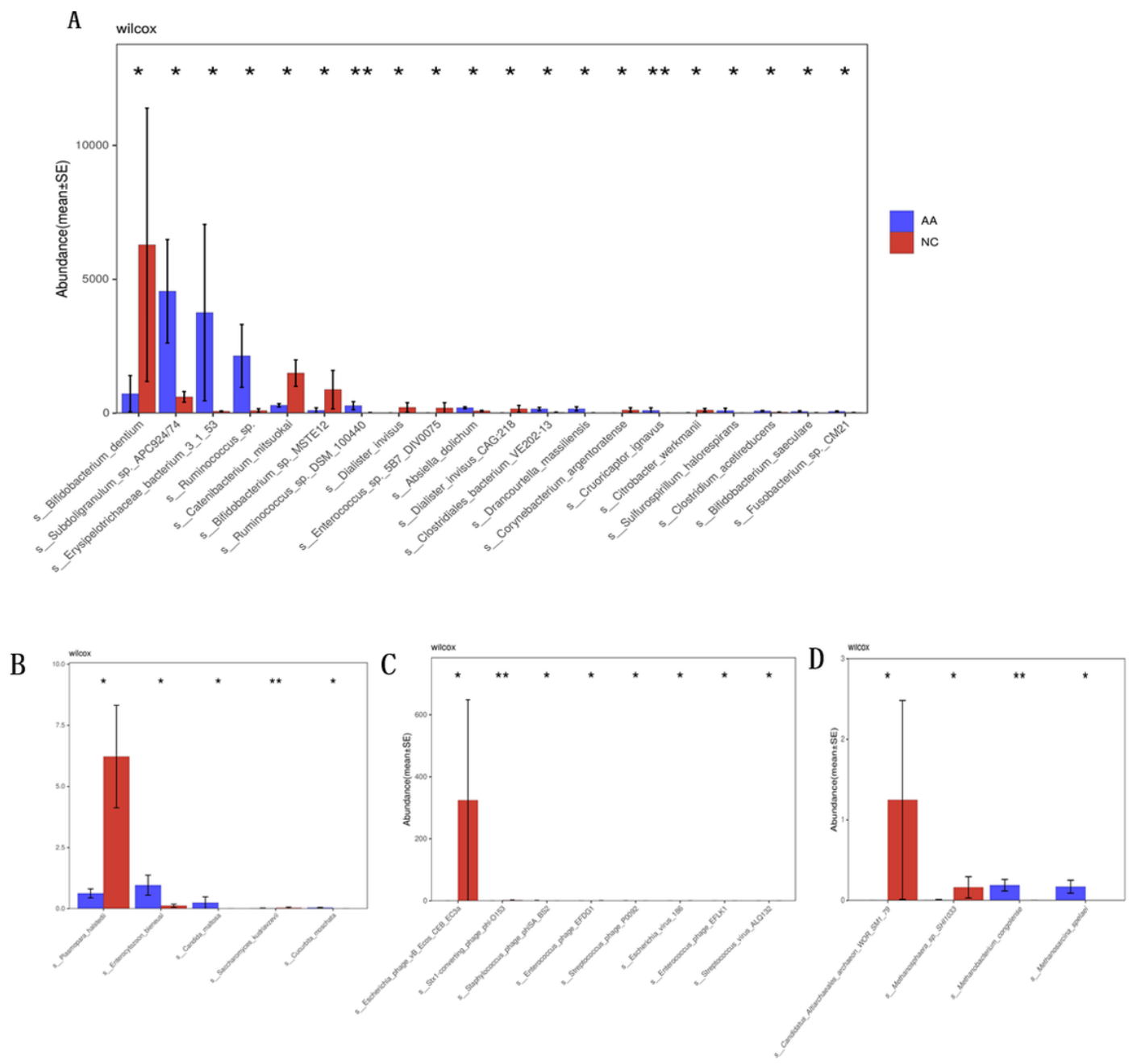

Figure 17

A. the changed bacterial abundance of AA patients in the species level. B. the changed eukaryote abundance of AA patients in the species level. C. the changed virus abundance of AA patients in the species level. D. the changed archaea abundance of AA patients in the species level. 


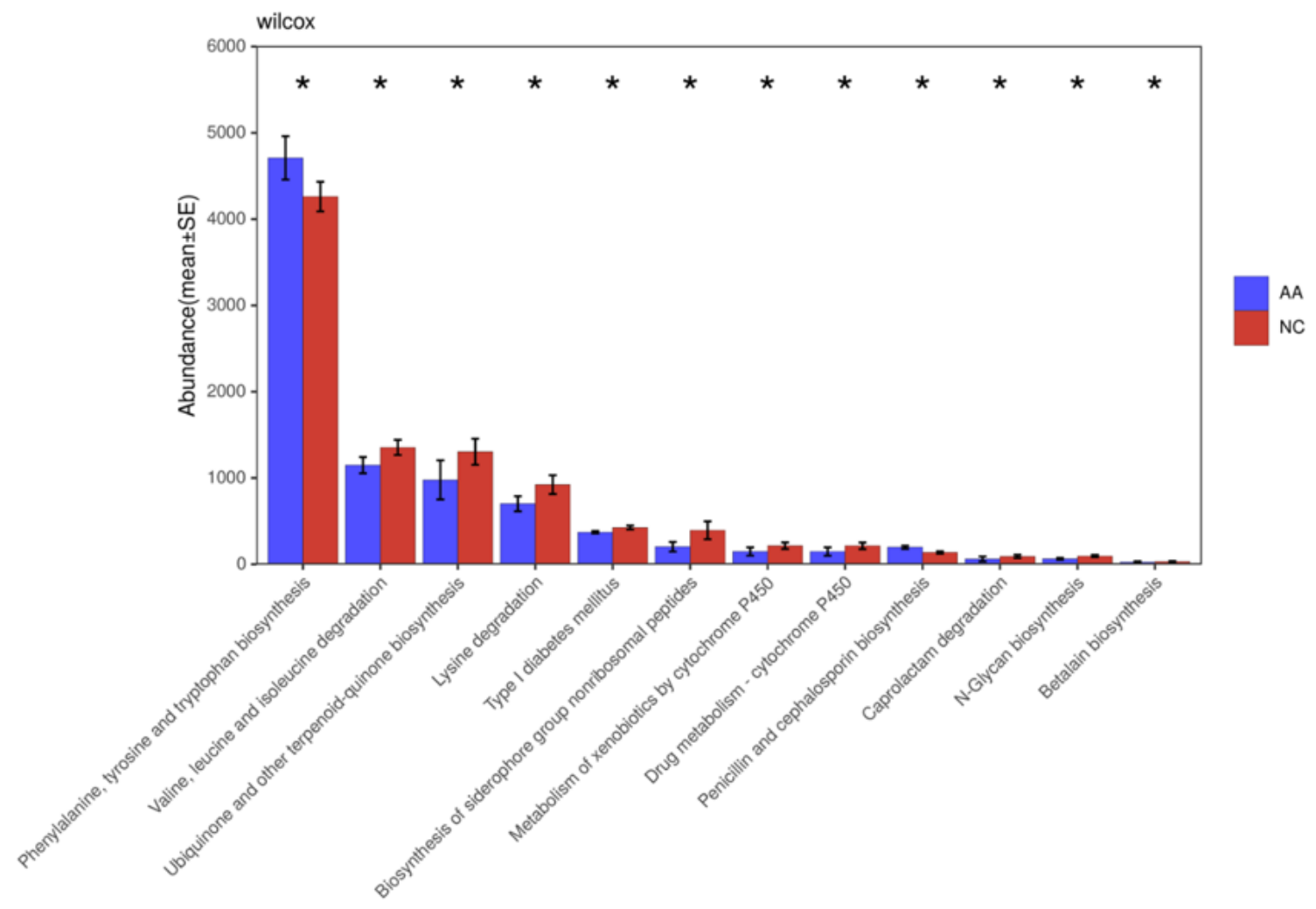

\section{Figure 18}

Changed abundance of KEGG pathway in AA group

A.

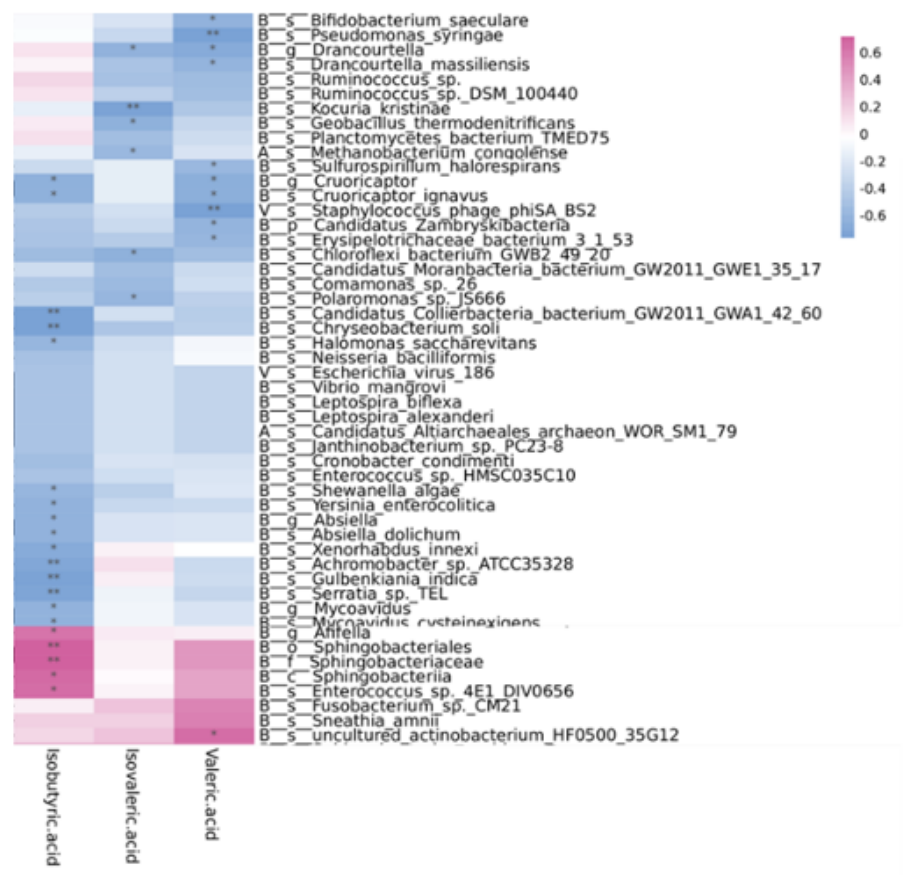

B.

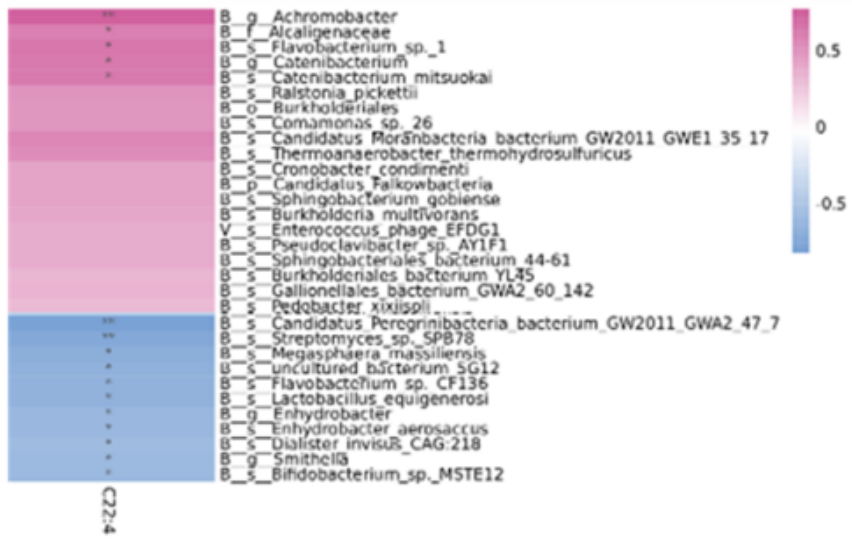

Figure 19 
A.the correlation between microbial abundance and short-chain fatty acids in the bone marrow supernatant in AA group. B. the correlation between microbial abundance and long-chain fatty acids in the bone marrow supernatant in AA group. (only the significant correlation showed here)

A

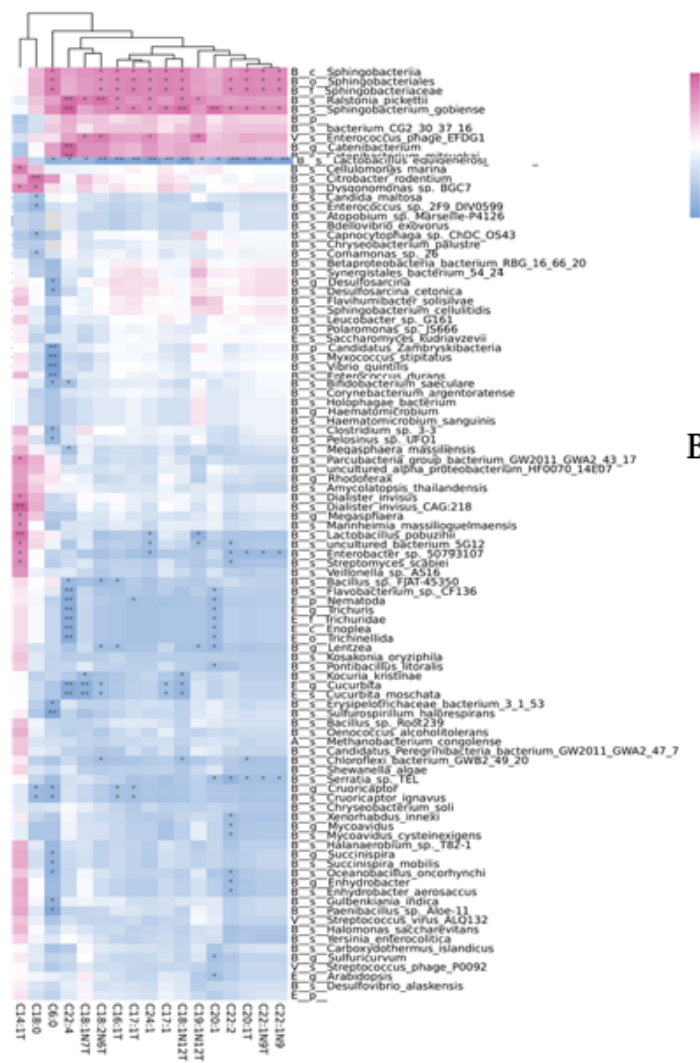

os

B

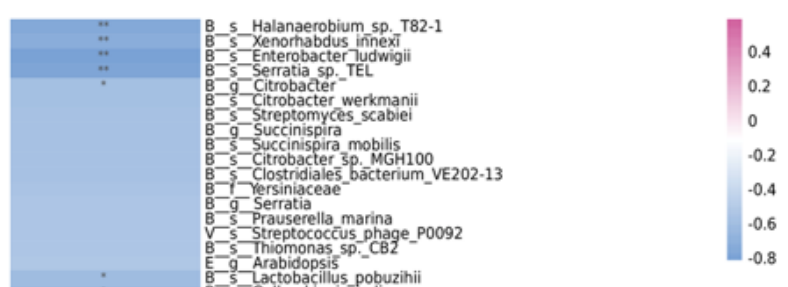

Figure 20

A. the correlation between microbial abundance and long-chain fatty acids in the plasm in AA group. B. the correlation between microbial abundance and ahort-chain fatty acids in the plasm in AA group. (only the significant correlation showed here) 

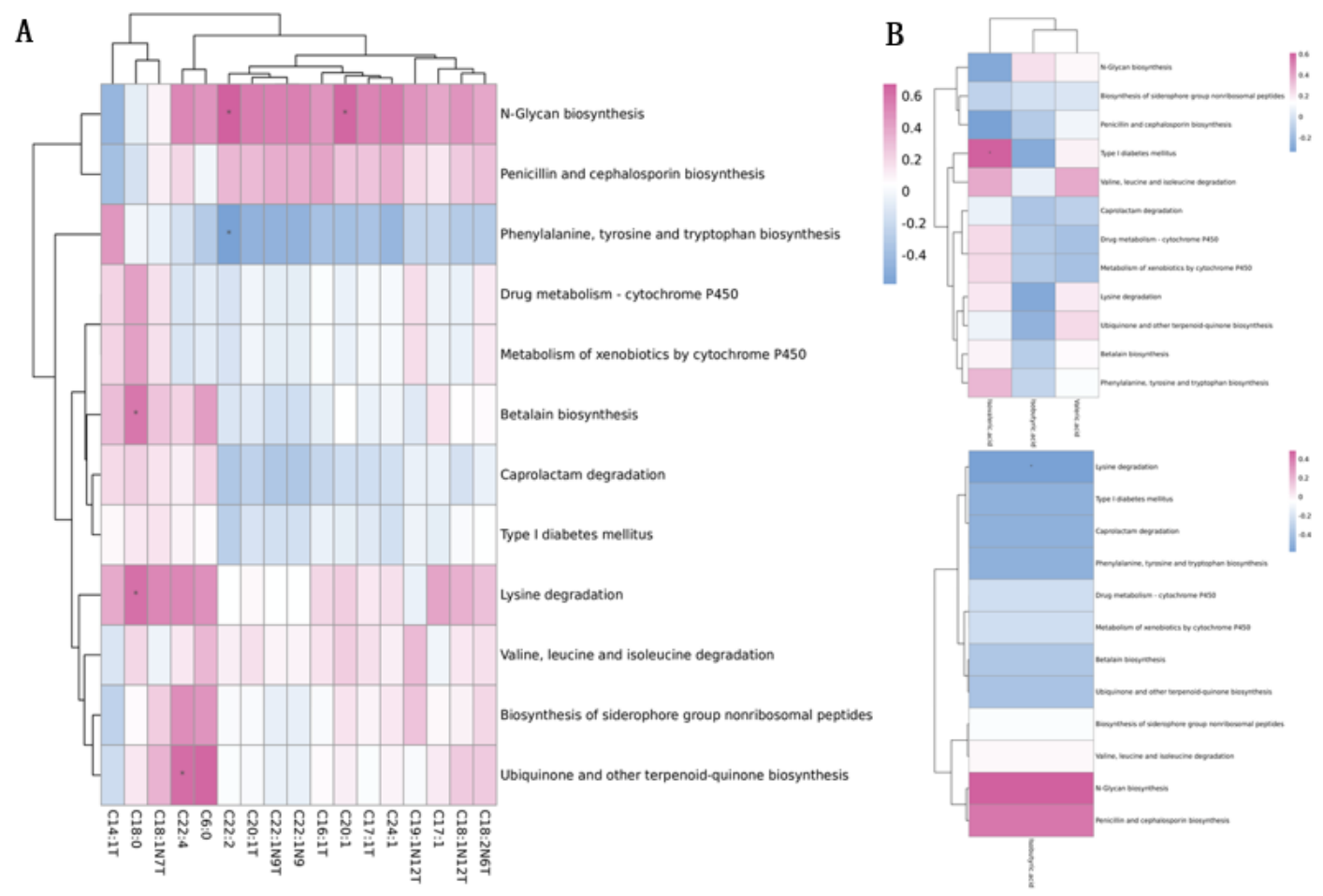

Figure 21

A.the correlation between the abundance of KEGG pathway and the long-chain fatty acids. B. the correlation between abundance of KEGG pathway and the short-chain fatty acids 


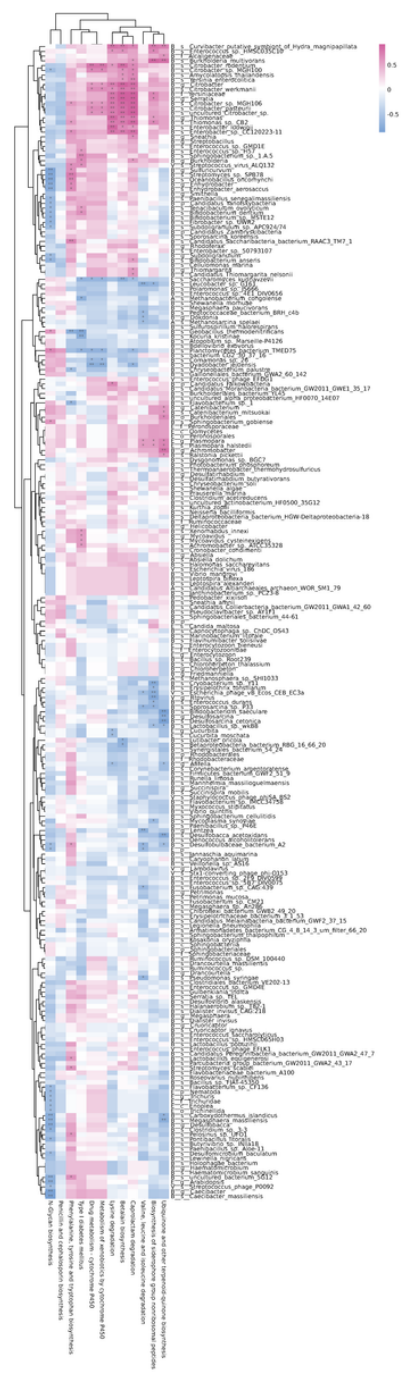

Figure 22

the correlation between the microbial abundance and the KEGG pathway abundance. 


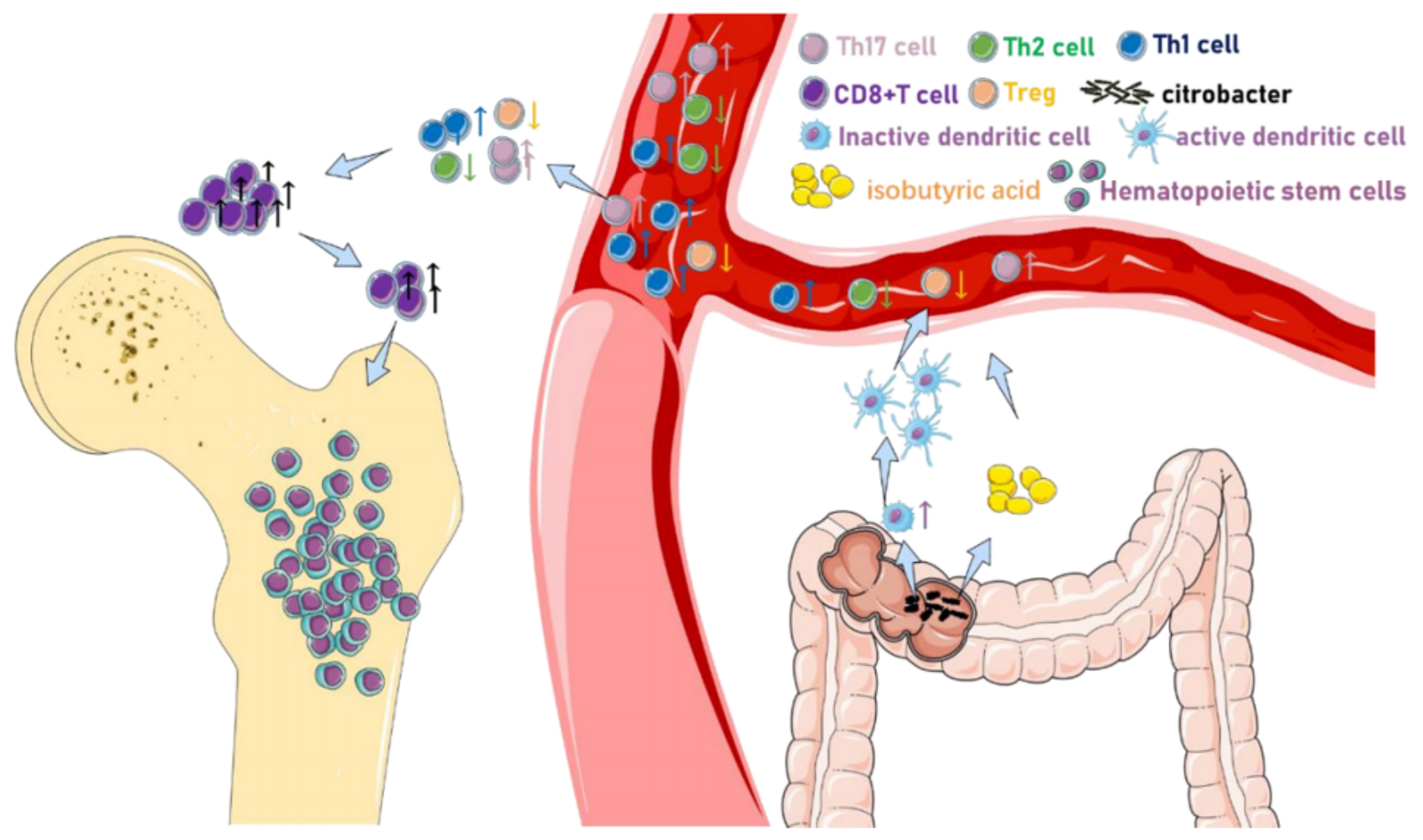

Figure 23

Citrobacter infection may affect the immune status of the body directly or by adjusting the content of isobutyric acid in the intestines, thereby inducing aplastic anemia. 